\title{
SECOND-ORDER AND STABILITY ANALYSIS FOR STATE-CONSTRAINED ELLIPTIC OPTIMAL CONTROL PROBLEMS WITH SPARSE CONTROLS*
}

\author{
EDUARDO CASAS $^{\dagger}$ AND FREDI TRÖLTZSCH ${ }^{\ddagger}$
}

\begin{abstract}
An optimal control problem for a semilinear elliptic partial differential equation is discussed subject to pointwise control constraints on the control and the state. The main novelty of the paper is the presence of the $L^{1}$-norm of the control as part of the objective functional that eventually leads to sparsity of the optimal control functions. Second-order sufficient optimality conditions are analyzed. They are applied to show the convergence of optimal solutions for vanishing $L^{2}$-regularization parameter for the control. The associated convergence rate is estimated.
\end{abstract}

Key words. optimal control, semilinear elliptic partial differential equation, pointwise state constraints, sparse control, first- and second-order optimality conditions, stability analysis

AMS subject classifications. 90C48, 49K20, 35J61

DOI. $10.1137 / 130917314$

1. Introduction. In this paper, we study the optimal control problem

$$
\left(\mathrm{P}_{\nu}\right) \quad \min _{u \in U_{a d}} J_{\nu}(u)
$$

where

$$
\begin{aligned}
J_{\nu}(u) & =\frac{1}{2} \int_{\Omega}\left(y_{u}(x)-y_{d}(x)\right)^{2} d x+\frac{\nu}{2} \int_{\Omega} u^{2}(x) d x+\kappa \int_{\Omega}|u(x)| d x, \\
U_{a d} & =\left\{u \in L^{\infty}(\Omega): \alpha \leq u(x) \leq \beta \text { for a.a. } x \in \Omega,\left|y_{u}(x)\right| \leq \gamma \forall x \in \Omega\right\},
\end{aligned}
$$

and $y_{u}$ is the solution of the Dirichlet problem

$$
\left\{\begin{aligned}
A y+a(x, y) & =u & & \text { in } \Omega, \\
y & =0 & & \text { on } \Gamma .
\end{aligned}\right.
$$

In this setting, $y_{d} \in L^{2}(\Omega)$ and real constants $\nu \geq 0, \kappa>0,-\infty<\alpha<0<$ $\beta<+\infty$, and $\gamma>0$ are given. The Dirichlet problem is considered in a bounded Lipschitz domain $\Omega \subset \mathbb{R}^{n}, n \in\{2,3\}$, with boundary $\Gamma=\partial \Omega$. Moreover, $A$ is a uniformly elliptic linear differential operator to be specified below, while $y_{d}$ is fixed in $L^{2}(\Omega)$.

Thanks to the presence of the $L^{1}$-norm in the objective functional, a convex but not differentiable functional is to be minimized. This term accounts for sparsity of optimal controls: With increasing parameter $\kappa$, the support of the optimal controls shrinks to finally have the measure zero for sufficiently large $\kappa$.

${ }^{*}$ Received by the editors April 17, 2013; accepted for publication (in revised form) January 2, 2014; published electronically March 20, 2014.

http://www.siam.org/journals/sicon/52-2/91731.html

†Departmento de Matemática Aplicada y Ciencias de la Computación, E.T.S.I. Industriales y de Telecomunicación, Universidad de Cantabria, 39005 Santander, Spain (eduardo.casas@unican.es). This author was supported by Spanish Ministerio de Economía y Competitividad under project MTM2011-22711.

¥Institut für Mathematik, Technische Universität Berlin, D-10623 Berlin, Germany (troeltzsch@ math.tu-berlin.de). This author was supported by the DFG Research Center MATHEON "Mathematics for key technologies" and the Collaborative Research Center SfB 910 Berlin.

1010 
Recently, such effects of sparsity attracted increasing interest. The first paper addressing this issue in the context of PDE control was [22], where the case of a linear elliptic equation was considered with the focus on numerical aspects. The associated analysis was extended in [25]. Later, the control of semilinear elliptic equations was investigated in [7] and [8]. For parabolic equations, sparsity can occur in different ways with respect to time and space variables. In particular, striped sparsity patterns (directional sparsity) can be obtained by suitable formulations of the cost functional. This type of sparsity was introduced in [19] in the context of linear elliptic and parabolic equations. Sparsity in time and space turned out to be important for (nonlinear) reaction-diffusion equations of Schlögl and FitzHugh-Nagumo type; cf. [12]. By admitting measures as controls instead of integrable control functions, the support of the optimal control can even have a zero Lebesgue measure; see [5], [6], and [14].

The main novelty of our contribution is the study of sparsity under the presence of pointwise state constraints, in particular the discussion of second-order sufficient optimality conditions and the convergence analysis with respect to a vanishing regularization parameter $\nu$. Second-order conditions were investigated for problems without sparsity in many papers; see the references in [24, p. 352]. For sparse control problems, the nondifferentiability of the objective functional causes new difficulties. This was addressed in [7] for the case without state constraints. Complementing the control constraints by state constraints in our paper further increases the level of difficulty. Moreover, our results even extend the whole theory of second-order conditions for state constrained problems. In particular, we allow for a vanishing Tikhonov parameter and compare various formulations of second-order conditions known in the literature.

We also discuss the stability of optimal solutions of the problems $\left(\mathrm{P}_{\nu}\right)$ for $\nu \rightarrow$ 0 . We show that, under a second-order sufficient optimality condition for $\left(\mathrm{P}_{0}\right)$, the optimal solutions of $\left(\mathrm{P}_{\nu}\right)$ converge strongly, and we estimate the order of convergence.

The plan of our paper is as follows: After stating the main assumptions in section 2.1 , we shall discuss the well-posedness of the optimal control problem and investigate the differentiability properties of the control-to-state mapping. In section 2.2 , we derive first-order necessary optimality conditions in a fairly standard way, while section 3 is devoted to second-order sufficient optimality conditions. Here, we distinguish between the cases $\nu>0$ and $\nu=0$. Finally, we perform a convergence analysis for the optimal control problem when $\nu$ tends to zero. The second-order and convergence analysis of sections 3 and 4 constitute the main novelty of our paper, although the first-order necessary optimality conditions are also new.

\section{Assumptions and preliminary results.}

2.1. Notation and main assumptions. Let us fix right here the following sets:

$$
\begin{aligned}
\mathcal{U}_{\alpha, \beta} & =\left\{u \in L^{\infty}(\Omega): \alpha \leq u(x) \leq \beta \text { for a.a. } x \in \Omega\right\}, \\
Y_{\gamma} & =\left\{y \in C_{0}(\Omega):|y(x)| \leq \gamma \forall x \in \Omega\right\} .
\end{aligned}
$$

Moreover, to shorten the notation, we introduce for $\nu \geq 0$ the family of functionals $F_{\nu}: L^{2}(\Omega) \rightarrow \mathbb{R}_{+}$and the functional $j: L^{2}(\Omega) \rightarrow \mathbb{R}_{+}$defined by

$$
\begin{aligned}
F_{\nu}(u) & :=\frac{1}{2} \int_{\Omega}\left(y_{u}(x)-y_{d}(x)\right)^{2} d x+\frac{\nu}{2} \int_{\Omega} u^{2}(x) d x, \\
j(u) & :=\int_{\Omega}|u(x)| d x
\end{aligned}
$$

Copyright (c) by SIAM. Unauthorized reproduction of this article is prohibited. 
so that we have

$$
J_{\nu}(u)=F_{\nu}(u)+\kappa j(u) .
$$

Notice that $j$ is convex and continuous but not differentiable.

On the state equation (1.2), we impose the following assumptions.

Assumption 1. A is the linear operator

$$
A y=-\sum_{i, j=1}^{n} \partial_{x_{j}}\left[a_{i j}(x) \partial_{x_{i}} y\right]
$$

with $a_{i j} \in L^{\infty}(\Omega)$, and there exists some $\Lambda>0$ such that

$$
\sum_{i, j=1}^{n} a_{i j}(x) \xi_{i} \xi_{j} \geq \Lambda|\xi|^{2} \text { for a.a. } x \in \Omega \text { and } \forall \xi \in \mathbb{R}^{n} .
$$

Assumption 2. $a: \Omega \times \mathbb{R} \longrightarrow \mathbb{R}$ is a Carathéodory function of class $C^{2}$ with respect to the second variable, with $a(\cdot, 0) \in L^{\bar{p}}(\Omega)$ for some $\bar{p}>\frac{n}{2}$, and satisfying

$$
\left\{\begin{array}{l}
\frac{\partial a}{\partial y}(x, y) \geq 0 \text { for a.a. } x \in \Omega \text { and } \forall y \in \mathbb{R} \\
\forall M>0 \exists C_{M}>0 \text { such that } \sum_{j=1}^{2}\left|\frac{\partial^{j} a}{\partial y^{j}}(x, y)\right| \leq C_{M} \text { for a.a. } x \in \Omega \text { and }|y| \leq M
\end{array}\right.
$$

Moreover, we assume some uniform continuity of $\frac{\partial^{2} a}{\partial y^{2}}$ : For every $M>0$ and $\varepsilon>0$, there exists $\rho_{\varepsilon, M}>0$ such that

$$
\left|\frac{\partial^{2} a}{\partial y^{2}}\left(x, y_{2}\right)-\frac{\partial^{2} a}{\partial y^{2}}\left(x, y_{1}\right)\right|<\varepsilon \quad \text { if }\left|y_{i}\right| \leq M \text { and }\left|y_{2}-y_{1}\right|<\rho_{\varepsilon, M} \text { for a.a. } x \in \Omega \text {. }
$$

We finish this section by recalling some known properties of the functional $j$. Since $j$ is convex and Lipschitz, the subdifferential in the sense of convex analysis and the generalized gradients introduced by Clarke coincide. Moreover, a simple computation shows that $\lambda \in \partial j(u)$ if and only if $\lambda$ is measurable and satisfies

$$
\begin{cases}\lambda(x)=+1 & \text { if } u(x)>0 \\ \lambda(x)=-1 & \text { if } u(x)<0 \\ \lambda(x) \in[-1,+1] & \text { if } u(x)=0\end{cases}
$$

holds a.e. in $\Omega$. Further, $j$ has directional derivatives given by

$$
j^{\prime}(u ; v)=\lim _{\rho \searrow 0} \frac{j(u+\rho v)-j(u)}{\rho}=\int_{\Omega_{u}^{+}} v d x-\int_{\Omega_{u}^{-}} v d x+\int_{\Omega_{u}^{0}}|v| d x
$$

for $u, v \in L^{1}(\Omega)$, where $\Omega_{u}^{+}, \Omega_{u}^{-}$, and $\Omega_{u}^{0}$ represent the sets of points where $u$ is positive, negative, or zero, respectively. Finally, the following relation holds:

$$
\max _{\lambda \in \partial j(u)} \int_{\Omega} \lambda v d x=j^{\prime}(u ; v) \leq \frac{j(u+\rho v)-j(u)}{\rho} \quad \forall 0<\rho \leq 1 .
$$

Copyright (C) by SIAM. Unauthorized reproduction of this article is prohibited. 
We refer to Clarke [15, Chapter 2] and Bonnans and Shapiro [2, section 2.4.3] for more details.

Remark 2.1. By $\left(\mathrm{P}_{\nu}\right)$, we discuss a simplified version of the control problem for better readability. Our theory can be extended to more general formulations by obvious modifications. This includes the case of state constraints of the type $\gamma_{1}(x) \leq y(x) \leq \gamma_{2}(x)$, where one of the equalities might be missing. Instead of assuming $\alpha<0$, sparsity can be also obtained for $\alpha=0$; see [12]. Notice that in many applications only nonnegative controls are meaningful. Finally, the more general cost functional

$$
F_{\nu}(u)=\int_{\Omega} L\left(x, y_{u}(x)\right) d x+\frac{\nu}{2} \int_{\Omega} u^{2}(x) d x+\kappa \int_{\Omega}|u(x)| d x
$$

can be treated in an analogous way; see [7].

2.2. Well-posedness of the optimal control problem and first-order optimality conditions. We start with known properties of the control-to-state mapping associated with the state equation (1.2).

TheOREM 2.2. Under Assumptions 1 and 2, to each $u \in L^{2}(\Omega)$ there exists a unique solution $y_{u} \in H_{0}^{1}(\Omega) \cap C(\bar{\Omega})$ of the state equation (1.2). The mapping $G: u \mapsto y_{u}$ is twice continuously Fréchet differentiable from $L^{2}(\Omega)$ to $H_{0}^{1}(\Omega) \cap C(\bar{\Omega})$. Its derivative $z_{v}:=G^{\prime}(u) v$ is given by the solution $z$ of

$$
\left\{\begin{aligned}
A z+\frac{\partial a}{\partial y}\left(x, y_{u}\right) z & =v & & \text { in } \Omega, \\
z & =0 & & \text { on } \Gamma,
\end{aligned}\right.
$$

while the second derivative $z_{v_{1}, v_{2}}:=G^{\prime \prime}(u)\left(v_{1}, v_{2}\right)$ associated with directions $v_{i} \in$ $L^{2}(\Omega), i=1,2$, is the solution $z$ of

$$
\left\{\begin{aligned}
A z+\frac{\partial a}{\partial y}\left(x, y_{u}\right) z & =-\frac{\partial^{2} a}{\partial y^{2}}\left(x, y_{u}\right) z_{v_{1}} z_{v_{2}} & & \text { in } \Omega, \\
z & =0 & & \text { on } \Gamma,
\end{aligned}\right.
$$

where the functions $z_{v_{i}}$ are defined by (2.4) above.

The proof of the existence, uniqueness, and regularity of $y_{u}$ is well known; see $[24$, section 4.2$]$ and the references therein. Let us show the differentiability of $G$. We set

$$
V=\left\{y \in H_{0}^{1}(\Omega): A y \in L^{q}(\Omega)\right\}
$$

with $q=\min \{2, \bar{p}\}$. Endowed with the graph norm, $V$ is a Banach space. Moreover, we deduce from [18, Theorem 8.30] that $V$ is embedded in $C(\bar{\Omega})$. Now, we consider

$$
\mathcal{F}: V \times L^{2}(\Omega) \longrightarrow L^{q}(\Omega), \quad \mathcal{F}(y, u):=A y+a(x, y)-u .
$$

Due to Assumption 2, $\mathcal{F}$ is well defined. Moreover, it is easy to check that $\mathcal{F}$ is of class $C^{2}, \mathcal{F}\left(y_{u}, u\right)=(0,0)$ for every $u \in L^{2}(\Omega)$, and

$$
\frac{\partial \mathcal{F}}{\partial y}\left(y_{u}, u\right): V \longrightarrow L^{q}(\Omega), \quad \frac{\partial \mathcal{F}}{\partial y}\left(y_{u}, u\right) z=A z+\frac{\partial a}{\partial y}\left(x, y_{u}\right) z
$$

defines an isomorphism. Now, the implicit function theorem yields that $G$ is of class $C^{2}$ and (2.4) and (2.5) are fulfilled.

Copyright $@$ by SIAM. Unauthorized reproduction of this article is prohibited. 
From Theorem 2.2 and the chain rule, we obtain the following differentiability property of $F_{\nu}$.

THEOREM 2.3. The functional $F_{\nu}: L^{2}(\Omega) \longrightarrow \mathbb{R}$ is of class $C^{2}$ and the first and second derivatives are given by

$$
\begin{aligned}
F_{\nu}^{\prime}(u) v & =\int_{\Omega}\left(\varphi_{u}+\nu u\right) v d x \\
F_{\nu}^{\prime \prime}(u)\left(v_{1}, v_{2}\right) & =\int_{\Omega}\left\{\left[1-\frac{\partial^{2} a}{\partial y^{2}}\left(x, y_{u}\right) \varphi_{u}\right] z_{v_{1}} z_{v_{2}}+\nu v_{1} v_{2}\right\} d x,
\end{aligned}
$$

where $z_{v_{i}}=G^{\prime}(u) v_{i}, i=1,2$, and $\varphi_{u} \in H_{0}^{1}(\Omega) \cap L^{\infty}(\Omega)$ is the adjoint state defined as the unique solution of

$$
\left\{\begin{aligned}
A^{*} \varphi+\frac{\partial a}{\partial y}\left(x, y_{u}\right) \varphi & =y_{u}-y_{d} & & \text { in } \Omega, \\
\varphi & =0 & & \text { on } \Gamma
\end{aligned}\right.
$$

with $A^{*}$ being the adjoint operator of $A$.

For (1.2), we know that if $u_{k} \rightarrow u$ weakly in $L^{2}(\Omega)$, then $G\left(u_{k}\right)=y_{u_{k}} \rightarrow y_{u}=$ $G(u)$ strongly in $H_{0}^{1}(\Omega) \cap C(\bar{\Omega})$. Indeed, by using a classical approach, see [23, Theorems 4.1 and 4.2], along with the monotonicity of $a(x, y)$ with respect to $y$, we can get a uniform bound for $\left\{y_{u_{k}}\right\}_{k}$ in $L^{\infty}(\Omega)$. Then, [18, Theorem 8.29] shows the boundedness of $\left\{y_{u_{k}}\right\}_{k}$ in a space of Hölder functions $C^{\theta}(\bar{\Omega})$ for some $0<\theta<1$. The compactness of the embedding $C^{\theta}(\bar{\Omega}) \subset C(\bar{\Omega})$ implies the convergence $y_{u_{k}} \rightarrow y_{u}$ in $C(\bar{\Omega})$. Finally, since the embedding $L^{2}(\Omega) \subset H^{-1}(\Omega)$ is compact, $u_{k} \rightarrow u$ strongly in $H^{-1}(\Omega)$, which allows us to conclude the strong convergence $y_{u_{k}} \rightarrow y_{u}$ in $H_{0}^{1}(\Omega)$. This property implies that $u \in U_{a d}$ if $\left\{u_{k}\right\}_{k} \subset U_{a d}$. Hence, by the continuity and convexity of the integrals in (1.1) involving the control, we get the following existence result in a standard way.

TheOREm 2.4. Let $U_{a d}$ be nonempty. Then, for every $\nu \geq 0$, problem $\left(P_{\nu}\right)$ has at least one optimal solution denoted by $u_{\nu}$.

Notice that $\left(\mathrm{P}_{\nu}\right)$ is a nonconvex problem, and hence more than one optimal solution might exist for fixed $\nu$. The assumption $U_{a d} \neq \emptyset$ is satisfied in particular if $a(x, 0)=0$ holds for a.a. $x \in \Omega$; then $u=0$ belongs to $U_{a d}$. Throughout the paper, we use the notation $y_{\nu}:=y_{u_{\nu}}$.

To establish first-order necessary optimality conditions of Karush-Kuhn-Tucker type, we assume the following linearized Slater condition.

Assumption 3 (linearized Slater condition). For given $\nu$, there exists $u_{s, \nu} \in \mathcal{U}_{\alpha, \beta}$ such that

$$
\left|y_{\nu}(x)+z_{u_{s, \nu}-u_{\nu}}(x)\right|<\gamma \forall x \in \Omega,
$$

where $z_{u_{s, \nu}-u_{\nu}}:=G^{\prime}\left(u_{\nu}\right)\left(u_{s, \nu}-u_{\nu}\right)$.

We shall prove later that this assumption is satisfied for all sufficiently small $\nu>0$ if it holds for $\nu=0$; see Theorem 4.3.

Let us now recall the first-order necessary optimality conditions for a given optimal solution $u_{\nu}$ which are a straightforward consequence of an abstract result proved by Bonnans and Casas in [1, Theorem 2.1].

TheOrem 2.5. Suppose that Assumption 3 holds. Let $u_{\nu}$ be optimal for $\left(\mathrm{P}_{\nu}\right)$ and let $y_{\nu}$ be the associated optimal state. Then there exist a regular Borel measure 
$\mu_{\nu}$, an adjoint state function $\varphi_{\nu} \in W_{0}^{1, s}(\Omega)$ for all $s<n /(n-1)$, and a function $\lambda_{\nu} \in \partial j\left(u_{\nu}\right) \subset L^{\infty}(\Omega)$ such that the following conditions are fulfilled:

$$
\begin{aligned}
& \left\{\begin{aligned}
A y_{\nu}+a\left(x, y_{\nu}\right) & =u_{\nu} & & \text { in } \Omega, \\
y_{\nu} & =0 & & \text { on } \Gamma
\end{aligned}\right. \\
& \left\{\begin{aligned}
A^{*} \varphi_{\nu}+\frac{\partial a}{\partial y}\left(x, y_{\nu}\right) \varphi_{\nu} & =y_{\nu}-y_{d}+\mu_{\nu} & & \text { in } \Omega, \\
\varphi_{\nu} & =0 & & \text { on } \Gamma,
\end{aligned}\right. \\
& \int_{\Omega}\left(y-y_{\nu}\right) d \mu_{\nu} \leq 0 \quad \forall y \in Y_{\gamma} \\
& \int_{\Omega} \lambda_{\nu}\left(u-u_{\nu}\right) d x+\int_{\Omega}\left|u_{\nu}\right| d x \leq \int_{\Omega}|u| d x \quad \forall u \in L^{1}(\Omega), \\
& \int_{\Omega}\left(\varphi_{\nu}+\nu u_{\nu}+\kappa \lambda_{\nu}\right)\left(u-u_{\nu}\right) d x \geq 0 \quad \forall u \in \mathcal{U}_{\alpha, \beta} .
\end{aligned}
$$

In this theorem, $(2.8)$ is the adjoint equation, $\mu_{\nu}$ denotes the Lagrange multiplier associated with the state constraint, (2.9) accounts for the complementary slackness condition, (2.10) expresses the subdifferential relation for $\partial j\left(u_{\nu}\right)$, and (2.11) is the variational inequality for $u_{\nu}$.

The next relations that we formulate for the cases $\nu>0$ and $\nu=0$ are immediate conclusions of (2.10) and (2.11).

Case $\nu>0$. There holds for a.a. $x \in \Omega$

$$
\begin{aligned}
& u_{\nu}(x)=\mathbb{P}_{[\alpha, \beta]}\left(-\frac{1}{\nu}\left[\varphi_{\nu}(x)+\kappa \lambda_{\nu}(x)\right]\right), \\
& u_{\nu}(x)=0 \text { iff }\left|\varphi_{\nu}(x)\right| \leq \kappa \\
& \lambda_{\nu}(x)=\mathbb{P}_{[-1,1]}\left(-\frac{1}{\kappa} \varphi_{\nu}(x)\right),
\end{aligned}
$$

where $\mathbb{P}_{[s, t]}: \mathbb{R} \rightarrow \mathbb{R}$ is the projection function on the interval $[s, t]$. We refer to Casas, Herzog, and Wachsmuth [7]. From (2.12) and (2.14) we deduce the following regularity results for $u_{\nu}$ and $\lambda_{\nu}$. In what follows, we denote by $M(\Omega)$ the Banach space of all real and regular Borel measures on $\Omega$.

THEOREM 2.6. Let $\left(u_{\nu}, y_{\nu}, \varphi_{\nu}, \mu_{\nu}, \lambda_{\nu}\right)$ satisfy the optimality system (2.7)-(2.11) with $u_{\nu} \in U_{a d}$ and $\nu>0$. Then, $u_{\nu}$ and $\lambda_{\nu}$ belong to $H_{0}^{1}(\Omega)$.

The proof of this theorem is based on the following result that is proved in $[16$, Theorem 10.1 and equation (2.22)]; see also [10].

Lemma 2.7. Given $\mu \in \mathcal{M}(\Omega)$, let $y \in W_{0}^{1, s}(\Omega)$ for all $s<n /(n-1)$ be the solution of

$$
\left\{\begin{aligned}
A y+a_{0} y=\mu & \text { in } \Omega, \\
y=0 & \text { on } \Gamma,
\end{aligned}\right.
$$

$a_{0} \geq 0$ belonging to $L^{\infty}(\Omega)$. Then, $\mathbb{P}_{[-M, M]}(y)$ belongs to $H_{0}^{1}(\Omega)$ for every $M>0$ and

$$
\Lambda\left\|\nabla \mathbb{P}_{[-M, M]}(y)\right\|_{L^{2}(\Omega)}^{2} \leq M\|\mu\|_{\mathcal{M}(\Omega)}
$$

Copyright (c) by SIAM. Unauthorized reproduction of this article is prohibited. 
Proof of Theorem 2.6. The regularity $\lambda_{\nu} \in H_{0}^{1}(\Omega)$ is an immediate consequence of Lemma 2.7 and (2.14). To establish the regularity of $u_{\nu}$ from identity (2.12), we cannot directly use Lemma 2.7 because $\varphi_{\nu}+\kappa \lambda_{\nu}$ is not necessarily a solution to an elliptic equation with a measure on the right-hand side. To overcome this difficulty we select $M>\kappa+\nu \max \{-\alpha, \beta\}$ and $\varphi_{\nu}^{M}(x)=\mathbb{P}_{[-M,+M]}\left(\varphi_{\nu}(x)\right)$. Then, once again Lemma 2.7 implies that $\varphi_{\nu}^{M} \in H_{0}^{1}(\Omega)$. From the definition of $M$ and (2.12) it is easy to check that

$$
\begin{aligned}
u_{\nu}(x) & =\mathbb{P}_{[\alpha, \beta]}\left(-\frac{1}{\nu}\left[\varphi_{\nu}(x)+\kappa \lambda_{\nu}(x)\right]\right) \\
& =\mathbb{P}_{[\alpha, \beta]}\left(-\frac{1}{\nu}\left[\varphi_{\nu}^{M}(x)+\kappa \lambda_{\nu}(x)\right]\right) \in H_{0}^{1}(\Omega) .
\end{aligned}
$$

For the limit case $\nu=0$, we introduce the following notation:

$$
\bar{u}:=u_{0}, \bar{\varphi}:=\varphi_{0}, \bar{y}:=y_{0}, \bar{\lambda}:=\lambda_{0}, \bar{\mu}:=\mu_{0} .
$$

Case $\nu=0$. Here, the following implications hold true for a.a. $x \in \Omega$ :

$$
\begin{aligned}
|\bar{\varphi}(x)| & <\kappa \Rightarrow \bar{u}(x)=0, \\
\bar{\varphi}(x) & >\kappa \Rightarrow \bar{u}(x)=\alpha, \\
\bar{\varphi}(x) & <-\kappa \Rightarrow \bar{u}(x)=\beta, \\
\bar{\varphi}(x) & =\kappa \Rightarrow \bar{u}(x) \leq 0, \\
\bar{\varphi}(x) & =-\kappa \Rightarrow \bar{u}(x) \geq 0, \\
\bar{\lambda}(x) & =\mathbb{P}_{[-1,1]}\left(-\frac{1}{\kappa} \bar{\varphi}(x)\right) .
\end{aligned}
$$

This result was shown in [4]. Using once again Lemma 2.7, we get that $\bar{\lambda} \in H_{0}^{1}(\Omega)$. From (2.9) we get the following well known property of $\mu_{\nu}$. If we consider the Jordan decomposition $\mu_{\nu}=\mu_{\nu}^{+}-\mu_{\nu}^{-}$, then we have

$$
\operatorname{supp}\left(\mu_{\nu}^{+}\right) \subset\left\{x \in \Omega: y_{\nu}(x)=+\gamma\right\} \text { and } \operatorname{supp}\left(\mu_{\nu}^{-}\right) \subset\left\{x \in \Omega: y_{\nu}(x)=-\gamma\right\} ;
$$

see, for instance, [3].

In what follows, we shall use for short the notation

$$
z_{s \nu}:=z_{u_{s, \nu}-u_{\nu}} .
$$

Next, we prove the boundedness of the adjoint states, uniform with respect to $\nu$ and an extra regularity property of $\mu_{\nu}$. The next theorem is inspired by a recent regularity result by Pieper and Vexler [21]; see also [9] for a posterior extension to the semilinear case. They considered the Poisson equation with measures as controls and observed that the optimal control enjoys $H^{-1}(\Omega)$ regularity. It became clear to us that we could extend this approach to analyze the regularity of the Lagrange multiplier $\mu_{\nu}$ and the corresponding adjoint state. The reader is also referred to [10], where the authors have obtained recently a similar result for more general pointwise state constraints and a linear state equation.

THEOREM 2.8. Let $\left(u_{\nu}, y_{\nu}, \varphi_{\nu}, \mu_{\nu}, \lambda_{\nu}\right)$ satisfy the optimality system (2.7)-(2.11) with $u_{\nu} \in U_{a d}$. Assume that $\alpha \leq a(x, 0) \leq \beta, a(x,+\gamma)>\alpha$, and $a(x,-\gamma)<\beta$ hold a.e. in $\Omega$. Then, there exists $M_{\nu}>0$ such that

$$
\left\|\varphi_{\nu}\right\|_{L^{\infty}(\Omega)}+\left\|\varphi_{\nu}\right\|_{H_{0}^{1}(\Omega)} \leq M_{\nu} .
$$

Moreover, $\mu_{\nu}$ is contained in $H^{-1}(\Omega) \cap M(\Omega)$. 
We prepare the proof of this theorem by some auxiliary results. The next result permits one to invoke certain regularity properties for the Laplace operator in investigating the Dirichlet problem for $A$.

LEMma 2.9. Let $\mu \in M(\Omega)$ be a positive measure with a compact support in $\Omega$ and $g_{A}$ be the Green's function corresponding to the Dirichlet problem associated with $A+a_{0} I, a_{0} \geq 0$ belonging to $L^{\infty}(\Omega)$. Define $y_{\mu}$ and $z_{\mu}$ as the solutions to the problems

$$
\left\{\begin{array} { r l } 
{ A y + a _ { 0 } y = \mu } & { \text { in } \Omega , } \\
{ y = 0 } & { \text { on } \Gamma }
\end{array} \quad \text { and } \quad \left\{\begin{array}{rl}
-\Delta z=\mu & \text { in } \Omega, \\
z=0 & \text { on } \Gamma .
\end{array}\right.\right.
$$

Then, for every $\varepsilon>0$ there exists a compact set $K_{\varepsilon}$ and a constant $C_{\varepsilon}>0$ such that $\operatorname{supp}(\mu) \subset K_{\varepsilon} \subset \Omega$ and

$$
\left\{\begin{array}{l}
\frac{1}{C_{\varepsilon}} z_{\mu}(x) \leq y_{\mu}(x) \leq C_{\varepsilon} z_{\mu}(x) \quad \text { for a.a. } x \in K_{\varepsilon}, \\
y_{\mu}(x)+z_{\mu}(x)<\varepsilon \quad \text { for a.a. } x \in \Omega \backslash K_{\varepsilon} .
\end{array}\right.
$$

In particular, it holds that $y_{\mu} \in L^{\infty}(\Omega)$ iff $z_{\mu} \in L^{\infty}(\Omega)$. Define further

$$
y_{\mu}^{*}(x):=\int_{\Omega} g_{A}(x, \xi) d \mu(\xi) \quad \forall x \in \Omega .
$$

Then it holds that $y_{\mu}^{*}(x)=y_{\mu}(x)$ a.e. in $\Omega$ and

$$
y_{\mu} \in L^{\infty}(\Omega) \Leftrightarrow \sup _{x \in \operatorname{supp}(\mu)} y_{\mu}^{*}(x)<\infty .
$$

Proof. First, let us observe that the function $y_{\mu}^{*}$ defined in the statement of the lemma is a particular function in the $L^{1}(\Omega)$-equivalence class of the solution $y_{\mu}$; see, for instance, [23, Theorem 9.4] or [20]. The same holds for $z_{\mu}$ and the function $z_{\mu}^{*}$ defined by

$$
z_{\mu}^{*}(x):=\int_{\Omega} g(x, \xi) d \mu(\xi) \quad \forall x \in \Omega,
$$

where $g$ is the Green's function in $\Omega$ associated with the operator $-\Delta$. Therefore, $y_{\mu}^{*}$ and $z_{\mu}^{*}$ are univocally defined at every point $x \in \Omega$, possibly being infinite at some points. However, $y_{\mu}$ and $z_{\mu}$ are only defined almost everywhere.

Observe that the positivity of $\mu$ implies that $y_{\mu}$ and $z_{\mu}$ are nonnegative almost everywhere in $\Omega$. Moreover, since $A y_{\mu}+a_{0} y_{\mu}=\Delta z_{\mu}=0$ in the open set $\Omega \backslash \operatorname{supp}(\mu)$ and $y_{\mu}=z_{\mu}=0$ on $\Gamma$, we deduce that $y_{\mu}, z_{\mu} \in C(\bar{\Omega} \backslash \operatorname{supp}(\mu))$. Therefore, given $\varepsilon>0$ we can choose a compact set $K_{\varepsilon}$ such that $\operatorname{supp}(\mu) \subset K_{\varepsilon} \subset \Omega$ and the second inequality of (2.22) holds. Let us prove the first inequality of (2.22). We know from $\left[23\right.$, p. 252] that there exists a positive number $C_{\varepsilon}$ such that

$$
\frac{1}{C_{\varepsilon}} g(x, y) \leq g_{A}(x, y) \leq C_{\varepsilon} g(x, y) \quad \forall x, y \in K_{\varepsilon} .
$$

Integration with respect to $\mu$ and taking into account that $\mu \geq 0$ and $\operatorname{supp}(\mu) \subset K_{\varepsilon}$ yields for all $x \in K_{\varepsilon}$

$$
\begin{aligned}
\frac{1}{C_{\varepsilon}} z_{\mu}^{*}(x) & =\frac{1}{C_{\varepsilon}} \int_{\Omega} g(x, \xi) d \mu(\xi)=\frac{1}{C_{\varepsilon}} \int_{K_{\varepsilon}} g(x, \xi) d \mu(\xi) \leq \int_{K_{\varepsilon}} g_{A}(x, \xi) d \mu(\xi) \\
& =\int_{\Omega} g_{A}(x, \xi) d \mu(\xi)=y_{\mu}^{*}(x) \leq C_{\varepsilon} \int_{K_{\varepsilon}} g(x, \xi) d \mu(\xi)=C_{\varepsilon} z_{\mu}^{*}(x) .
\end{aligned}
$$

These inequalities imply the first inequality of (2.22).

Copyright $\odot$ by SIAM. Unauthorized reproduction of this article is prohibited. 
By the first part of our proof, we have

$$
y_{\mu} \in L^{\infty}(\Omega) \Leftrightarrow z_{\mu} \in L^{\infty}(\Omega) \Leftrightarrow \sup _{x \in \operatorname{supp}(\mu)} z_{\mu}^{*}(x)<\infty,
$$

where the last equivalence is due to a result by Pieper and Vexler [21].

Finally, the inequalities

$$
\frac{1}{C_{\varepsilon}} z_{\mu}^{*}(x) \leq y_{\mu}^{*}(x) \leq C_{\varepsilon} z_{\mu}^{*}(x) \quad \forall x \in \operatorname{supp}(\mu) \subset K_{\varepsilon}
$$

and the above equivalences imply that

$$
\sup _{x \in \operatorname{supp}(\mu)} y_{\mu}^{*}(x)<\infty \Leftrightarrow \sup _{x \in \operatorname{supp}(\mu)} z_{\mu}^{*}(x)<\infty \Leftrightarrow z_{\mu} \in L^{\infty}(\Omega) \Leftrightarrow y_{\mu} \in L^{\infty}(\Omega) .
$$

Lemma 2.10. Let $\mu \in M(\Omega)$ be given and let $\mu=\mu^{+}-\mu^{-}$be its Jordan decomposition. Assume that there exist compact and disjoint sets $K^{+}$and $K^{-}$, both contained in $\Omega$, such that $\operatorname{supp} \mu^{+} \subset K^{+}$and $\operatorname{supp} \mu^{-} \subset K^{-}$. Let $y_{\mu}$ be the unique solution of

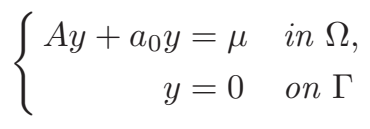

and denote analogously by $y_{\mu^{+}}, y_{\mu^{-}}, y_{|\mu|}$ the solution of (2.23) with right-hand side $\mu^{+}, \mu^{-},|\mu|$, respectively. Then we have the following implications:

(i) $y_{\mu} \in L^{\infty}(\Omega) \Leftrightarrow y_{\mu^{+}}$and $y_{\mu^{-}} \in L^{\infty}(\Omega) \Leftrightarrow y_{|\mu|} \in L^{\infty}(\Omega)$.

(ii) If $y_{\mu}$ belongs to $L^{\infty}(\Omega)$, then it holds that $y_{\mu} \in H_{0}^{1}(\Omega)$ and $\mu$ belongs to $H^{-1}(\Omega)$. Moreover, the following inequality holds:

$$
\Lambda\left\|\nabla y_{\mu}\right\|_{L^{2}(\Omega)}^{2} \leq\left\|y_{\mu}\right\|_{L^{\infty}(\Omega)}\|\mu\|_{M(\Omega)} .
$$

Proof. (i) Assume $y_{\mu} \in L^{\infty}(\Omega)$. We fix disjoint open subsets $\Omega_{+}, \Omega_{-}$of $\Omega$ such that $K_{+} \subset \Omega_{+}$and $K_{-} \subset \Omega_{-}$. Then a result by Stampacchia [23, Theorem 9.3 and its proof] yields $y_{\mu^{+}} \in C\left(\overline{\Omega_{-}}\right)$and $y_{\mu^{-}} \in C\left(\overline{\Omega_{+}}\right)$. We obtain

$$
\left\|y_{\mu^{+}}\right\|_{L^{\infty}\left(\Omega_{+}\right)} \leq\left\|y_{\mu}\right\|_{L^{\infty}\left(\Omega_{+}\right)}+\left\|y_{\mu^{-}}\right\|_{L^{\infty}\left(\Omega_{+}\right)}<\infty,
$$

because the first term is bounded by our assumption and the second is also bounded thanks to the result by Stampacchia quoted above. Therefore, $y_{\mu^{+}}$belongs to $L^{\infty}\left(\Omega_{+}\right)$. By Lemma 2.9, we even have $y_{\mu^{+}} \in L^{\infty}(\Omega)$. In the same way, one shows $y_{\mu^{-}} \in L^{\infty}(\Omega)$. The remaining statements of (i) are obvious.

(ii) We set $K:=\left\|y_{\mu}\right\|_{L^{\infty}(\Omega)}$; then Lemma 2.7 implies

$$
y_{\mu}(x)=\mathbb{P}_{[-K, K]}\left(y_{\mu}(x)\right) \in H_{0}^{1}(\Omega),
$$

and (2.24) holds. Now it is obvious that $\mu$ belongs to $H^{-1}(\Omega)$.

Proof of Theorem 2.8. Let us write $\varphi_{\nu}=\varphi_{\nu}^{0}+\varphi_{\nu}^{+}-\varphi_{\nu}^{-}$, where $\varphi_{\nu}^{0}, \varphi_{\nu}^{+}$and $\varphi_{\nu}^{-}$are the solutions of the adjoint equation (2.8) with right-hand side equal to $y_{\nu}-y_{d}, \mu_{\nu}^{+}$, and $\mu_{\nu}^{-}$, respectively. Since $y_{\nu}-y_{d} \in L^{2}(\Omega)$, we know that $\varphi_{\nu}^{0} \in H_{0}^{1}(\Omega) \cap C(\bar{\Omega})$. We will prove that $\varphi_{\nu}^{+}$and $\varphi_{\nu}^{-}$belong to $L^{\infty}(\Omega)$. First, we observe that both functions are nonnegative. Thus, we only need to prove that they are bounded from above.

Copyright $@$ by SIAM. Unauthorized reproduction of this article is prohibited. 
Let us define

$$
\Omega_{\nu}^{+}:=\left\{x \in \Omega: y_{\nu}(x)>\frac{\gamma}{2}\right\}, \quad \Omega_{\nu}^{-}:=\left\{x \in \Omega: y_{\nu}(x)<-\frac{\gamma}{2}\right\} .
$$

Recall that $\operatorname{supp}\left(\mu^{+}\right) \subset \Omega_{\nu}^{+}$and $\operatorname{supp}\left(\mu^{-}\right) \subset \Omega_{\nu}^{-}$. Then, following Stampacchia [23, Theorem 9.3 and proof], we know that $\varphi_{\nu}^{-} \in C\left(\overline{\Omega_{\nu}^{+}}\right), \varphi_{\nu}^{+} \in C\left(\overline{\Omega_{\nu}^{-}}\right)$, and

$$
\exists K_{\gamma}>0 \text { such that } \max \left(\left\|\varphi_{\nu}^{-}\right\|_{C\left(\overline{\Omega_{\nu}^{+}}\right)},\left\|\varphi_{\nu}^{+}\right\|_{C\left(\overline{\Omega_{\nu}^{-}}\right)}\right) \leq K_{\gamma}\left\|\mu_{\nu}\right\|_{M(\Omega)} .
$$

We shall prove below that

$$
\operatorname{ess} \sup _{x \in \Omega} \varphi_{\nu}^{+}(x) \leq M_{\nu}^{+}:=\max (1, \nu)|\alpha|+K_{\gamma}\left\|\mu_{\nu}\right\|_{M(\Omega)}+\kappa+\left\|\varphi_{\nu}^{0}\right\|_{L^{\infty}(\Omega)} .
$$

Then we have

$$
\varphi_{\nu}(x) \leq \varphi_{\nu}^{0}(x)+\varphi_{\nu}^{+}(x) \leq\left\|\varphi_{\nu}^{0}\right\|_{L^{\infty}(\Omega)}+M_{\nu}^{+} \quad \text { a.e. in } \Omega .
$$

Analogously, we deduce

$$
\text { ess } \sup _{x \in \Omega} \varphi_{\nu}^{-}(x) \leq M_{\nu}^{-}:=\max (1, \nu)|\beta|+K_{\gamma}\left\|\mu_{\nu}\right\|_{M(\Omega)}+\kappa+\left\|\varphi_{\nu}^{0}\right\|_{L^{\infty}(\Omega)},
$$

and hence

$$
\varphi_{\nu}(x) \geq \varphi_{\nu}^{0}(x)-\varphi_{\nu}^{-}(x) \geq-\left\|\varphi_{\nu}^{0}\right\|_{L^{\infty}(\Omega)}-M_{\nu}^{-} \quad \text { a.e. in } \Omega .
$$

Altogether, we obtain the desired $L^{\infty}$-bound

$$
\left\|\varphi_{\nu}\right\|_{L^{\infty}(\Omega)} \leq M_{\nu}:=\max \left(M_{\nu}^{+}, M_{\nu}^{-}\right)+\left\|\varphi_{\nu}^{0}\right\|_{L^{\infty}(\Omega)}
$$

The estimate for $\varphi_{\nu}$ in $H_{0}^{1}(\Omega)$ follows from (2.24). The regularity of $\mu_{\nu}$ is a consequence of Lemma 2.10.

Therefore, it remains to prove (2.25). We proceed by contradiction and assume that $\varphi_{\nu}^{+}$is not bounded from above by $M_{\nu}^{+}$. Define

$$
\varphi_{\nu}^{*+}(x)=\int_{\Omega} g_{A}(x, \xi) d \mu_{\nu}^{+}(\xi) \text { and } \varphi_{\nu}^{*-}(x)=\int_{\Omega} g_{A}(x, \xi) d \mu_{\nu}^{-}(\xi),
$$

and $\varphi_{\nu}^{*}=\varphi_{\nu}^{0}+\varphi_{\nu}^{*+}-\varphi_{\nu}^{*-}$. It follows from Lemma 2.9 that there exists $x_{0} \in \operatorname{supp} \mu_{\nu}^{+}$ such that

$$
\varphi_{\nu}^{*+}\left(x^{0}\right)>M_{\nu}^{+}
$$

The function $\varphi_{\nu}^{*+}$ is lower semicontinuous. This follows from Fatou's lemma and the integral representation of $\varphi_{\nu}^{*+}$. (Indeed, as any Green's function, $g_{A}$ is nonnegative and continuous for $x \neq \xi$ and $\lim _{x^{\prime} \rightarrow x} g_{A}\left(x^{\prime}, x\right)=g_{A}(x, x)=+\infty$.) In view of this, there exists a $\rho>0$ such that

$$
\varphi_{\nu}^{*+}(x)>M_{\nu}^{+} \quad \forall x \in \bar{B}_{\rho}\left(x^{0}\right) \text { and } \bar{B}_{\rho}\left(x^{0}\right) \subset \Omega_{\nu}^{+} .
$$

This is possible due to

$$
\operatorname{supp} \mu_{\nu}^{+} \subset\left\{x \in \Omega: y_{\nu}(x)=\gamma\right\}
$$

Copyright (c) by SIAM. Unauthorized reproduction of this article is prohibited. 
This implies

$$
\begin{aligned}
\varphi_{\nu}^{*}(x) & =\varphi_{\nu}^{0}(x)+\varphi_{\nu}^{*+}(x)-\varphi_{\nu}^{*-}(x)>-\left\|\varphi_{\nu}^{0}\right\|_{L^{\infty}(\Omega)}+M_{\nu}^{+}-\sup _{x \in \bar{\Omega}_{\nu}^{+}} \varphi_{\nu}^{*}(x) \\
& \geq-\left\|\varphi_{\nu}^{0}\right\|_{L^{\infty}(\Omega)}+M_{\nu}^{+}-K_{\gamma}\left\|\mu_{\nu}\right\|_{M(\Omega)}=\max (1, \nu)|\alpha|+\kappa \quad \forall x \in \bar{B}_{\rho}\left(x^{0}\right) .
\end{aligned}
$$

Therefore, since $\varphi_{\nu}(x)=\varphi_{\nu}^{*}(x)$ holds for almost all $x \in \Omega$ and $\left\|\lambda_{\nu}\right\|_{L^{\infty}(\Omega)} \leq 1$, we have

$$
\varphi_{\nu}(x)+\kappa \lambda_{\nu}(x)>\max (1, \nu)|\alpha| \quad \text { for a.a. } x \in \bar{B}_{\rho}\left(x^{0}\right) .
$$

Now we distinct between the cases $\nu>0$ and $\nu=0$.

Case $\nu>0$. Here, it holds that

$$
u_{\nu}(x)=\mathbb{P}_{[\alpha, \beta]}\left(-\frac{1}{\nu}\left[\varphi_{\nu}(x)+\kappa \lambda_{\nu}(x)\right]\right)=\alpha,
$$

and we are able to continue by

$$
A y_{\nu}=u_{\nu}-a(x, 0)+[a(x, 0)-a(x, y)] \leq \alpha-a(x, 0) \leq 0 \quad \text { a.e. in } \bar{B}_{\rho}\left(x^{0}\right) .
$$

Notice that $a(x, 0) \geq \alpha$ holds by assumption and that $y_{\nu}$ is positive in $\Omega_{\nu}^{+}$. From the maximum principle for elliptic equations, we deduce

$$
\gamma=y_{\nu}\left(x_{0}\right) \leq \max _{x \in \bar{B}_{\rho}\left(x^{0}\right)}=\max _{x \in \partial B_{\rho}\left(x^{0}\right)} y_{\nu}(x) \leq \gamma,
$$

and then $y_{\nu}(x) \equiv \gamma$ in $\bar{B}_{\rho}\left(x^{0}\right)$. Hence, we have

$$
a(x, \gamma)=A y_{\nu}+a\left(x, y_{\nu}\right)=u_{\nu}=\alpha \quad \text { in } B_{\rho}\left(x^{0}\right),
$$

which contradicts the fact that $\alpha<a(x, \gamma)$ in $\Omega$.

Case $\nu=0$. We have

$$
\varphi_{\nu}(x)>|\alpha|+\kappa \quad \text { for a.e. } x \in \bar{B}_{\rho}\left(x^{0}\right) .
$$

Consequently, (2.16) leads to $u_{\nu}(x)=\alpha$ a.e. in $B_{\rho}\left(x^{0}\right)$. Then, we have again

$$
A y_{\nu} \leq \alpha-a(x, 0) \leq 0 \quad \text { a.e. in } B_{\rho}\left(x^{0}\right),
$$

and we continue in the same way as above to get the contradiction.

3. Second-order sufficient optimality conditions. In order to perform the second-order analysis of the control problem $\left(\mathrm{P}_{\nu}\right)$, we introduce the Lagrangian function $\mathcal{L}_{\nu}: L^{2}(\Omega) \times M(\Omega) \rightarrow \mathbb{R}$ defined by

$$
\mathcal{L}_{\nu}(u, \mu):=F_{\nu}(u)+\int_{\Omega} y_{u} d \mu .
$$

From Theorems 2.2 and 2.3, we obtain that $\mathcal{L}$ is of class $C^{2}$ and

$$
\begin{aligned}
\frac{\partial \mathcal{L}_{\nu}}{\partial u}(u, \mu) v & =F_{\nu}^{\prime}(u) v+\int_{\Omega} z_{v} d \mu=\int_{\Omega}\left(\varphi_{u}+\nu u\right) v d x, \\
\frac{\partial^{2} \mathcal{L}_{\nu}}{\partial u^{2}}(u, \mu) v^{2} & =\int_{\Omega}\left\{\left[1-\frac{\partial^{2} a}{\partial y^{2}}\left(x, y_{u}\right) \varphi_{u}\right] z_{v}^{2}+\nu v^{2}\right\} d x,
\end{aligned}
$$

Copyright (c) by SIAM. Unauthorized reproduction of this article is prohibited. 
where $\varphi_{u} \in W_{0}^{1, s}(\Omega)$ for all $s<\frac{n}{n-1}$ is the solution of

$$
\left\{\begin{aligned}
A^{*} \varphi+\frac{\partial a}{\partial y}\left(x, y_{u}\right) \varphi & =y_{u}-y_{d}+\mu & & \text { in } \Omega, \\
\varphi & =0 & & \text { on } \Gamma .
\end{aligned}\right.
$$

According to (3.2), the variational inequality (2.11) can be written in the form

$$
\frac{\partial \mathcal{L}_{\nu}}{\partial u}\left(u_{\nu}, \mu_{\nu}\right)\left(u-u_{\nu}\right)+\kappa \int_{\Omega} \lambda_{\nu}\left(u-u_{\nu}\right) d x \geq 0 \quad \forall u \in U_{\alpha, \beta} .
$$

Moreover, from (2.2) we also have

$$
\frac{\partial \mathcal{L}_{\nu}}{\partial u}\left(u_{\nu}, \mu_{\nu}\right)\left(u-u_{\nu}\right)+\kappa j^{\prime}\left(u_{\nu} ; u-u_{\nu}\right) d x \geq 0 \quad \forall u \in U_{\alpha, \beta} .
$$

In this section, $u_{\nu}$ will denote an element of $U_{a d}$ satisfying with $\left(y_{\nu}, \varphi_{\nu}, \lambda_{\nu}, \mu_{\nu}\right)$ the optimality system (2.7)-(2.11). Associated with $u_{\nu}$, we introduce the following cone of critical directions for every $\tau \geq 0$ :

$$
\begin{aligned}
& C_{u_{\nu}}^{\tau}=\left\{v \in L^{2}(\Omega):\right. \\
& \frac{\partial \mathcal{L}_{\nu}}{\partial u}\left(u_{\nu}, \mu_{\nu}\right) v+\kappa j^{\prime}\left(u_{\nu} ; v\right) \leq \tau\|v\|_{L^{2}(\Omega)}, \\
& v(x)\left\{\begin{array}{lll}
\geq 0 & \text { if } & u_{\nu}(x)=\alpha, \\
\leq 0 & \text { if } & u_{\nu}(x)=\beta,
\end{array}\right. \\
& z_{v}(x)\left\{\begin{array}{rll}
\leq \tau\|v\|_{L^{2}(\Omega)} & \text { if } & y_{\nu}(x)=\gamma, \\
\geq-\tau\|v\|_{L^{2}(\Omega)} & \text { if } & y_{\nu}(x)=-\gamma,
\end{array}\right. \\
& \left.\int_{\Omega}\left|z_{v}\right| d\left|\mu_{\nu}\right| \leq \tau\|v\|_{L^{2}(\Omega)}\right\} .
\end{aligned}
$$

In the case $\tau=0$, we simply write $C_{u_{\nu}}$ instead of $C_{u_{\nu}}^{0}$. As proved in [7, Lemma 3.5], if $v \in L^{2}(\Omega)$ satisfies (3.8), then

$$
\frac{\partial \mathcal{L}_{\nu}}{\partial u}\left(u_{\nu}, \mu_{\nu}\right) v+\kappa j^{\prime}\left(u_{\nu} ; v\right) \geq \frac{\partial \mathcal{L}_{\nu}}{\partial u}\left(u_{\nu}, \mu_{\nu}\right) v+\kappa \int_{\Omega} \lambda_{\nu} v d x \geq 0 .
$$

As a consequence of this, for $\tau=0$ we have

$$
\begin{aligned}
C_{u_{\nu}}=\left\{v \in L^{2}(\Omega):\right. & \\
& \frac{\partial \mathcal{L}_{\nu}}{\partial u}\left(u_{\nu}, \mu_{\nu}\right) v+\kappa j^{\prime}\left(u_{\nu} ; v\right)=0, \\
& v(x)\left\{\begin{array}{lll}
\geq 0 & \text { if } & u_{\nu}(x)=\alpha, \\
\leq 0 & \text { if } & u_{\nu}(x)=\beta,
\end{array}\right. \\
& z_{v}(x)\left\{\begin{array}{lll}
\leq 0 & \text { if } & y_{\nu}(x)=+\gamma, \\
\geq 0 & \text { if } & y_{\nu}(x)=-\gamma,
\end{array}\right. \\
& \left.\int_{\Omega}\left|z_{v}\right| d\left|\mu_{\nu}\right|=0\right\} .
\end{aligned}
$$

In the second-order analysis, we will distinguish between two cases depending on whether the parameter $\nu$ is strictly positive or zero.

Copyright (C) by SIAM. Unauthorized reproduction of this article is prohibited. 
3.1. Case $\boldsymbol{\nu}>\mathbf{0}$. The role played by the Tikhonov regularization term in the cost functional is crucial for the second-order analysis; see [11] for $\kappa=0$. Indeed, surprisingly some formulations of the second-order sufficient optimality conditions are equivalent, which is not true for general optimization problems in infinite dimension. In particular, this is not true for $\nu=0$. The next theorem states these equivalent formulations.

THEOREM 3.1. The following statements are equivalent:

$$
\begin{gathered}
\frac{\partial^{2} \mathcal{L}_{\nu}}{\partial u^{2}}\left(u_{\nu}, \mu_{\nu}\right) v^{2}>0 \quad \forall v \in C_{u_{\nu}} \backslash\{0\} \\
\exists \sigma>0 \text { and } \exists \tau>0 \text { such that } \frac{\partial^{2} \mathcal{L}_{\nu}}{\partial u^{2}}\left(u_{\nu}, \mu_{\nu}\right) v^{2} \geq \sigma\|v\|_{L^{2}(\Omega)}^{2} \quad \forall v \in C_{u_{\nu}}^{\tau}, \\
\exists \sigma>0 \text { and } \exists \tau>0 \text { such that } \frac{\partial^{2} \mathcal{L}_{\nu}}{\partial u^{2}}\left(u_{\nu}, \mu_{\nu}\right) v^{2} \geq \sigma\left\|z_{v}\right\|_{L^{2}(\Omega)}^{2} \quad \forall v \in C_{u_{\nu}}^{\tau},
\end{gathered}
$$

where $z_{v}=G^{\prime}\left(u_{\nu}\right) v$.

Proof. From the inequality $\left\|z_{v}\right\|_{L^{2}(\Omega)} \leq C\|v\|_{L^{2}(\Omega)}$, it is obvious that (3.17) implies (3.18) with the same $\tau$ and replacing $\sigma$ by $\sigma / C^{2}$ in (3.18). The implication $(3.18) \Rightarrow(3.16)$ is obvious. To prove $(3.16) \Rightarrow(3.17)$, we proceed by contradiction. We assume that (3.16) holds but (3.17) is false. Then, for every integer $k \geq 1$, there exists an element $v_{k} \in C_{u_{\nu}}^{1 / k}$ such that

$$
\frac{\partial^{2} \mathcal{L}_{\nu}}{\partial u^{2}}\left(u_{\nu}, \mu_{\nu}\right) v_{k}^{2}<\frac{1}{k}\left\|v_{k}\right\|_{L^{2}(\Omega)}^{2} .
$$

We divide $v_{k}$ by its $L^{2}(\Omega)$-norm and, selecting a subsequence if necessary, we obtain an element $v \in L^{2}(\Omega)$ such that

$$
\left\|v_{k}\right\|_{L^{2}(\Omega)}=1, \quad \frac{\partial^{2} \mathcal{L}_{\nu}}{\partial u^{2}}\left(u_{\nu}, \mu_{\nu}\right) v_{k}^{2}<\frac{1}{k} \quad \forall k \geq 1, \quad \text { and } \quad v_{k} \rightarrow v \text { in } L^{2}(\Omega) .
$$

Let us prove that $v \in C_{u_{\nu}}$. First we observe that $v$ satisfies (3.13) because every $v_{k}$ does it. Now, since the functional $L^{2}(\Omega) \ni v \mapsto j^{\prime}\left(u_{\nu} ; v\right) \in \mathbb{R}$ is convex and continuous, and $v_{k}$ satisfies (3.7) with $\tau=1 / k$, we can pass to the limit below, see (3.2), and deduce

$$
\frac{\partial \mathcal{L}_{\nu}}{\partial u}\left(u_{\nu}, \mu_{\nu}\right) v+\kappa j^{\prime}\left(u_{\nu} ; v\right) \leq \liminf _{k \rightarrow \infty}\left\{\frac{\partial \mathcal{L}_{\nu}}{\partial u}\left(u_{\nu}, \mu_{\nu}\right) v_{k}+\kappa j^{\prime}\left(u_{\nu} ; v_{k}\right)\right\} \leq 0 .
$$

This inequality and (3.11) imply that (3.12) holds for $v$. Moreover, the weak convergence $v_{k} \rightarrow v$ implies the strong convergence $z_{v_{k}} \rightarrow z_{v}$ in $C_{0}(\Omega)$. Therefore, it is easy to pass to the limit in (3.9) and (3.10) for $v_{k}$ and $\tau=1 / k$ and to obtain (3.14) and (3.15). This completes the proof of $v \in C_{u_{\nu}}$. On the other hand, we can pass to the limit in (3.19), see (3.3), and get

$$
\frac{\partial^{2} \mathcal{L}_{\nu}}{\partial u^{2}}\left(u_{\nu}, \mu_{\nu}\right) v^{2} \leq \liminf _{k \rightarrow \infty} \frac{\partial^{2} \mathcal{L}_{\nu}}{\partial u^{2}}\left(u_{\nu}, \mu_{\nu}\right) v_{k}^{2} \leq 0 .
$$

According to the assumption (3.16), this is only possible if $v=0$. This implies that $z_{v_{k}} \rightarrow 0$ strongly in $C_{0}(\Omega)$. Hence, using again the expressions (3.3) and (3.19), it follows that

$$
\nu=\lim _{k \rightarrow 0} \frac{\partial^{2} \mathcal{L}_{\nu}}{\partial u^{2}}\left(u_{\nu}, \mu_{\nu}\right) v_{k}^{2} \leq 0 .
$$

Since $\nu>0$, we have a contradiction. 
Theorem 3.2. Assume that $u_{\nu}$ satisfies (3.16). Then there exist $\delta>0$ and $\varepsilon>0$ such that

$$
J_{\nu}\left(u_{\nu}\right)+\frac{\delta}{2}\left\|u-u_{\nu}\right\|_{L^{2}(\Omega)}^{2} \leq J_{\nu}(u) \quad \forall u \in U_{a d} \cap B_{\varepsilon}\left(u_{\nu}\right),
$$

where $B_{\varepsilon}\left(u_{\nu}\right)$ denotes the $L^{2}(\Omega)$-ball centered at $u_{\nu}$ with radius $\varepsilon$.

Proof. Let us fix $\tau>0$ and $\sigma>0$ such that (3.17) holds. We prove this theorem by contradiction and assume that there exists a sequence $\left\{u_{k}\right\}_{k=1}^{\infty} \subset U_{a d}$ with

$$
\left\|u_{k}-u_{\nu}\right\|_{L^{2}(\Omega)}<\frac{1}{k} \text { and } J_{\nu}\left(u_{k}\right)<J_{\nu}\left(u_{\nu}\right)+\frac{1}{2 k}\left\|u_{k}-u_{\nu}\right\|_{L^{2}(\Omega)}^{2} .
$$

We shall show the existence of $k_{\tau}>0$ such that there holds

$$
u_{k}-u_{\nu} \in C_{u_{\nu}}^{\tau} \quad \forall k \geq k_{\tau},
$$

i.e., $u_{k}-u_{\nu}$ belongs to the critical cone for all sufficiently large $k$. For this purpose, we have to confirm the conditions (3.7)-(3.10). To verify (3.7), we observe first that (2.9) implies

$$
\int_{\Omega}\left(y_{k}-y_{\nu}\right) d \mu_{\nu} \leq 0 \quad \forall k \geq 1 .
$$

By a Taylor expansion, it follows from (3.21) and (2.3) that

$$
\begin{aligned}
\frac{1}{2 k}\left\|u_{k}-u_{\nu}\right\|_{L^{2}(\Omega)}^{2}> & J_{\nu}\left(u_{k}\right)-J_{\nu}\left(u_{\nu}\right) \geq \mathcal{L}_{\nu}\left(u_{k}, \mu_{\nu}\right)-\mathcal{L}_{\nu}\left(u_{\nu}, \mu_{\nu}\right)+\kappa\left(j\left(u_{k}\right)-j\left(u_{\nu}\right)\right) \\
\geq & \frac{\partial \mathcal{L}_{\nu}}{\partial u}\left(u_{\nu}, \mu_{\nu}\right)\left(u_{k}-u_{\nu}\right)+\kappa j^{\prime}\left(u_{\nu} ; u_{k}-u_{\nu}\right) \\
& +\frac{1}{2} \frac{\partial^{2} \mathcal{L}_{\nu}}{\partial u^{2}}\left(u_{\nu}+\vartheta_{k}\left(u_{k}-u_{\nu}\right), \mu_{\nu}\right)\left(u_{k}-u_{\nu}\right)^{2}
\end{aligned}
$$

with some $\vartheta_{k} \in(0,1)$. This implies

$$
\begin{aligned}
& \frac{\partial \mathcal{L}}{\partial u}\left(u_{\nu}, \mu_{\nu}\right)\left(u_{k}-u_{\nu}\right)+\kappa j^{\prime}\left(u_{\nu} ; u_{k}-u_{\nu}\right) \\
& \quad \leq C\left\|u_{k}-u_{\nu}\right\|_{L^{2}(\Omega)}^{2} \leq \frac{C}{k}\left\|u_{k}-u_{\nu}\right\|_{L^{2}(\Omega)}<\tau\left\|u_{k}-u_{\nu}\right\|_{L^{2}(\Omega)}
\end{aligned}
$$

if $k>C / \tau$. Thus, the inequality (3.7) holds. It is obvious that condition (3.8) is satisfied for $v=u_{k}-u_{\nu}$.

Next, let us verify condition (3.9). We have the equations

$$
\begin{aligned}
A\left(y_{k}-y_{\nu}\right)+\frac{\partial a}{\partial y}\left(x, y_{\nu}\right)\left(y_{k}-y_{\nu}\right) & =u_{k}-u_{\nu}-\frac{1}{2} \frac{\partial^{2} a}{\partial y^{2}}\left(x, y_{\nu}+\vartheta_{k}\left(y_{k}-y_{\nu}\right)\right)\left(y_{k}-y_{\nu}\right)^{2}, \\
A z_{u_{k}-u_{\nu}}+\frac{\partial a}{\partial y}\left(x, y_{\nu}\right) z_{u_{k}-u_{\nu}} & =u_{k}-u_{\nu}
\end{aligned}
$$

for some measurable function $\vartheta_{k}: \Omega \rightarrow(0,1)$. Define $w_{k} \in H_{0}^{1}(\Omega)$ by

$$
A w_{k}+\frac{\partial a}{\partial y}\left(x, y_{\nu}\right) w_{k}=-\frac{1}{2} \frac{\partial^{2} a}{\partial y^{2}}\left(x, y_{\nu}+\vartheta_{k}\left(y_{k}-y_{\nu}\right)\right)\left(y_{k}-y_{\nu}\right)^{2} .
$$

Copyright $@$ by SIAM. Unauthorized reproduction of this article is prohibited. 
Then it holds that

$$
\left\|w_{k}\right\|_{L^{\infty}(\Omega)} \leq C\left\|y_{k}-y_{\nu}\right\|_{L^{\infty}(\Omega)}^{2} \leq C\left\|u_{k}-u_{\nu}\right\|_{L^{2}(\Omega)}^{2} .
$$

If $y_{\nu}(x)=\gamma$, then we have

$$
0 \geq y_{k}-y_{\nu}=z_{u_{k}-u_{\nu}}+w_{k}
$$

and it follows for $k>C / \tau$

$$
z_{u_{k}-u_{\nu}}(x) \leq-w_{k}(x) \leq \frac{C}{k}\left\|u_{k}-u_{\nu}\right\|_{L^{2}(\Omega)} \leq \tau\left\|u_{k}-u_{\nu}\right\|_{L^{2}(\Omega)} .
$$

This is the upper condition of (3.9). The lower one is verified in the same way.

It remains to check (3.10). Using again (3.21) and a Taylor expansion, we get with the help of $(2.3)$

$F_{\nu}^{\prime}\left(u_{\nu}\right)\left(u_{k}-u_{\nu}\right)+\frac{1}{2} F_{\nu}^{\prime \prime}\left(u_{\nu}+\vartheta_{k}\left(u_{k}-u_{\nu}\right)\right)\left(u_{k}-u_{\nu}\right)^{2}+\kappa j^{\prime}\left(u_{\nu} ; u_{k}-u_{\nu}\right) \leq \frac{1}{k}\left\|u_{k}-u_{\nu}\right\|_{L^{2}(\Omega)}^{2}$ for some $\vartheta_{k} \in(0,1)$. Therefore

$$
F_{\nu}^{\prime}\left(u_{\nu}\right)\left(u_{k}-u_{\nu}\right)+\kappa j^{\prime}\left(u_{\nu} ; u_{k}-u_{\nu}\right) \leq C\left\|u_{k}-u_{\nu}\right\|_{L^{2}(\Omega)}^{2} .
$$

Now, we proceed as follows:

$$
\begin{aligned}
& \int_{\Omega}\left|z_{u_{k}-u_{\nu}}\right| d\left|\mu_{\nu}\right|=\int_{\Omega}\left|z_{u_{k}-u_{\nu}}\right| d \mu_{\nu}^{+}+\int_{\Omega}\left|z_{u_{k}-u_{\nu}}\right| d \mu_{\nu}^{-} \\
& =\int_{\substack{z_{u_{k}-u_{\nu}>0}>0 \\
y_{\nu}=\gamma}} z_{u_{k}-u_{\nu}} d \mu_{\nu}^{+}-\int_{\substack{z_{u_{k}-u_{\nu}<0}<0 \\
y_{\nu}=-\gamma}} z_{u_{k}-u_{\nu}} d \mu_{\nu}^{-} \\
& -\int_{\substack{z_{u_{k}-u_{\nu}<0}<0 \\
y_{\nu}=\gamma}} z_{u_{k}-u_{\nu}} d \mu_{\nu}^{+}+\int_{\substack{z_{u_{k}-u_{\nu}>0}>0 \\
y_{\nu}=-\gamma}} z_{u_{k}-u_{\nu}} d \mu_{\nu}^{-} \\
& \leq \frac{C}{k}\left\|\mu_{\nu}^{+}\right\|_{M(\Omega)}\left\|u_{k}-u_{\nu}\right\|_{L^{2}(\Omega)}+\frac{C}{k}\left\|\mu_{\nu}^{-}\right\|_{M(\Omega)}\left\|u_{k}-u_{\nu}\right\|_{L^{2}(\Omega)} \\
& -\int_{\substack{z_{k}-u_{\nu}<0 \\
y_{\nu}=\gamma}} z_{u_{k}-u_{\nu}} d \mu_{\nu}^{+}+\int_{\substack{z_{k}-u_{\nu}>0 \\
y_{\nu}=-\gamma}} z_{u_{k}-u_{\nu}} d \mu_{\nu}^{-} \\
& \leq \frac{C}{k}\left\|\mu_{\nu}\right\|_{M(\Omega)}\left\|u_{k}-u_{\nu}\right\|_{L^{2}(\Omega)}-\int_{\substack{z_{u_{k}-u_{\nu}}<0 \\
y_{\nu}=\gamma}} z_{u_{k}-u_{\nu}} d \mu_{\nu}^{+} \\
& +\int_{\substack{u_{k}-u_{\nu}>0 \\
y_{\nu}=-\gamma}} z_{u_{k}-u_{\nu}} d \mu_{\nu}^{-} .
\end{aligned}
$$

In the last estimates, we have used inequality (3.9) with $\tau \leq \frac{C}{k}$ as proved in (3.22). We observe that, due to $u_{k} \in \mathcal{U}_{\alpha, \beta}$, (3.6) leads to

$$
\frac{\partial \mathcal{L}_{\nu}}{\partial u}\left(u_{\nu}, \mu_{\nu}\right)\left(u_{k}-u_{\nu}\right)+\kappa j^{\prime}\left(u_{\nu} ; u_{k}-u_{\nu}\right) \geq 0 .
$$

According to the first identity in (3.2) with $v=u_{k}-u_{\nu}$, the derivative of $\mathcal{L}_{\nu}$ contains the term

$$
\begin{aligned}
\int_{\Omega} z_{u_{k}-u_{\nu}} d \mu_{\nu}= & \int_{\substack{z_{u_{k}-u_{\nu}>0}>0 \\
y_{\nu}=\gamma}} z_{u_{k}-u_{\nu}} d \mu_{\nu}^{+}-\int_{\substack{z_{u_{k}-u_{\nu}<0}<0 \\
y_{\nu}=-\gamma}} z_{u_{k}-u_{\nu}} d \mu_{\nu}^{-} \\
& +\int_{\substack{z_{u_{k}-u_{\nu}}<0 \\
y_{\nu}=\gamma}} z_{u_{k}-u_{\nu}} d \mu_{\nu}^{+}-\int_{\substack{z_{u_{k}-u_{\nu}>0}>0 \\
y_{\nu}=-\gamma}} z_{u_{k}-u_{\nu}} d \mu_{\nu}^{-} .
\end{aligned}
$$

Copyright (c) by SIAM. Unauthorized reproduction of this article is prohibited. 
We move the last two terms to the other side of (3.24) and use (3.23) to deduce

$$
\begin{gathered}
-\iint_{\substack{z_{u_{k}-u_{\nu}}<0 \\
y_{\nu}=\gamma}} z_{u_{k}-u_{\nu}} d \mu_{\nu}^{+}+\int_{\substack{z_{u_{k}-u_{\nu}>0}>0 \\
y_{\nu}=-\gamma}} z_{u_{k}-u_{\nu}} d \mu_{\nu}^{-} \leq \frac{C}{k}\left\|\mu_{\nu}\right\|_{M(\Omega)}\left\|u_{k}-u_{\nu}\right\|_{L^{2}(\Omega)} \\
+F^{\prime}\left(u_{\nu}\right)\left(u_{k}-u_{\nu}\right)+\kappa j^{\prime}\left(u_{\nu} ; u_{k}-u_{\nu}\right) \leq \frac{C}{k}\left\|u_{k}-u_{\nu}\right\|_{L^{2}(\Omega) .}
\end{gathered}
$$

This shows (3.10), and altogether we have shown $u_{k}-u_{\nu} \in C_{u_{\nu}}^{\tau}$ for $k$ large enough.

Next, we derive the contradiction to our initial hypotheses. We proved above that

$$
\begin{aligned}
\frac{1}{2 k}\left\|u_{k}-u_{\nu}\right\|_{L^{2}(\Omega)}^{2}> & \frac{\partial \mathcal{L}_{\nu}}{\partial u}\left(u_{\nu}, \mu_{\nu}\right)\left(u_{k}-u_{\nu}\right)+\kappa j^{\prime}\left(u_{\nu} ; u_{k}-u_{\nu}\right) \\
& +\frac{1}{2} \frac{\partial^{2} \mathcal{L}_{\nu}}{\partial u^{2}}\left(u_{\nu}+\vartheta_{k}\left(u_{k}-u_{\nu}\right), \mu_{\nu}\right)\left(u_{k}-u_{\nu}\right)^{2}
\end{aligned}
$$

With (3.6) we obtain

$$
\frac{1}{2} \frac{\partial^{2} \mathcal{L}_{\nu}}{\partial u^{2}}\left(u_{k}+\vartheta_{k}\left(u_{k}-u_{\nu}\right), \mu_{\nu}\right)\left(u_{k}-u_{\nu}\right)^{2}<\frac{1}{2 k}\left\|u_{k}-u_{\nu}\right\|_{L^{2}(\Omega)}^{2} .
$$

We rewrite the left-hand side of this inequality and apply (3.17) to deduce

$$
\begin{gathered}
\frac{1}{2} \frac{\partial^{2} \mathcal{L}_{\nu}}{\partial u^{2}}\left(u_{k}+\vartheta_{k}\left(u_{k}-u_{\nu}\right), \mu_{\nu}\right)\left(u_{k}-u_{\nu}\right)^{2} \\
=\frac{1}{2} \frac{\partial^{2} \mathcal{L}_{\nu}}{\partial u^{2}}\left(u_{\nu}, \mu_{\nu}\right)\left(u_{k}-u_{\nu}\right)^{2} \\
+\frac{1}{2}\left[\frac{\partial^{2} \mathcal{L}_{\nu}}{\partial u^{2}}\left(u_{k}+\vartheta_{k}\left(u_{k}-u_{\nu}\right), \mu_{\nu}\right)-\frac{\partial^{2} \mathcal{L}_{\nu}}{\partial u^{2}}\left(u_{\nu}, \mu_{\nu}\right)\right]\left(u_{k}-u_{\nu}\right)^{2} \\
\geq \frac{\sigma}{2}\left\|u_{k}-u_{\nu}\right\|_{L^{2}(\Omega)}^{2}+\mathrm{I},
\end{gathered}
$$

where

$$
\mathrm{I}=\int_{\Omega}\left[1-\varphi_{\vartheta_{k}} \frac{\partial^{2} a}{\partial y^{2}}\left(x, y_{\vartheta_{k}}\right)\right] z_{\vartheta_{k}}^{2} d x-\int_{\Omega}\left[1-\varphi_{\nu} \frac{\partial^{2} a}{\partial y^{2}}\left(x, y_{\nu}\right)\right] z_{u_{k}-u_{\nu}}^{2} d x
$$

where $y_{\vartheta_{k}}$ and $\varphi_{\vartheta_{k}}$ denote the state and adjoint state associated with $u_{\nu}+\vartheta_{k}\left(u_{k}-u_{\nu}\right)$, and $z_{\vartheta_{k}}=G^{\prime}\left(u_{\nu}+\vartheta_{k}\left(u_{k}-u_{\nu}\right)\right)\left(u_{k}-u_{\nu}\right)$. To proceed with our estimation, we consider the following equations:

$$
\begin{aligned}
A z_{\vartheta_{k}}+\frac{\partial a}{\partial y}\left(x, y_{\vartheta_{k}}\right) z_{\vartheta_{k}} & =u_{k}-u_{\nu}, \\
A z_{u_{k}-u_{\nu}}+\frac{\partial a}{\partial y}\left(x, y_{\nu}\right) z_{u_{k}-u_{\nu}} & =u_{k}-u_{\nu} .
\end{aligned}
$$

Subtracting the two equations, we obtain

$$
A\left(z_{\vartheta_{k}}-z_{u_{k}-u_{\nu}}\right)+\frac{\partial a}{\partial y}\left(x, y_{\nu}\right)\left(z_{\vartheta_{k}}-z_{u_{k}-u_{\nu}}\right)=-\frac{\partial^{2} a}{\partial y^{2}}\left(x, \hat{y}_{\vartheta_{k}}\right)\left(y_{\vartheta_{k}}-y_{\nu}\right) z_{\vartheta_{k}}
$$

with $\hat{y}_{\vartheta_{k}}$ being intermediate functions between $y_{\vartheta_{k}}$ and $y_{\nu}$. Therefore, it follows that

$$
\begin{aligned}
\left\|z_{\vartheta_{k}}-z_{u_{k}-u_{\nu}}\right\|_{L^{2}(\Omega)} & \leq c\left\|y_{\vartheta_{k}}-y_{\nu}\right\|_{L^{\infty}(\Omega)}\left\|z_{\vartheta_{k}}\right\|_{L^{2}(\Omega)} \\
& \leq c\left\|u_{k}-u_{\nu}\right\|_{L^{2}(\Omega)}\left\|z_{\vartheta_{k}}\right\|_{L^{2}(\Omega)} \leq \frac{c}{k}\left\|z_{\vartheta_{k}}\right\|_{L^{2}(\Omega)}
\end{aligned}
$$

Copyright $@$ ㅇ by SIAM. Unauthorized reproduction of this article is prohibited. 
and

$$
\left\|z_{\vartheta_{k}}\right\|_{L^{2}(\Omega)} \leq\left\|z_{\vartheta_{k}}-z_{u_{k}-u_{\nu}}\right\|_{L^{2}(\Omega)}+\left\|z_{u_{k}-u_{\nu}}\right\|_{L^{2}(\Omega)} \leq \frac{c}{k}\left\|z_{\vartheta_{k}}\right\|_{L^{2}(\Omega)}+\left\|z_{u_{k}-u_{\nu}}\right\|_{L^{2}(\Omega)} .
$$

This estimate yields for $k>c$

$$
\left\|z_{\vartheta_{k}}\right\|_{L^{2}(\Omega)} \leq\left(1-\frac{c}{k}\right)^{-1}\left\|z_{u_{k}-u_{\nu}}\right\|_{L^{2}(\Omega)} .
$$

Next, we estimate the term I defined in (3.26). To this aim, we first consider the term $z_{\nu}^{2}-z_{u_{k}-u_{\nu}}^{2}:$

$$
\begin{aligned}
\int_{\Omega}\left|z_{\vartheta_{k}}^{2}-z_{u_{k}-u_{\nu}}^{2}\right| d x & \leq\left\|z_{\vartheta_{k}}+z_{u_{k}-u_{\nu}}\right\|_{L^{2}(\Omega)}\left\|z_{\vartheta_{k}}-z_{u_{k}-u_{\nu}}\right\|_{L^{2}(\Omega)} \\
& \leq \frac{c}{k}\left(\left\|z_{\vartheta_{k}}\right\|_{L^{2}(\Omega)}+\left\|z_{u_{k}-u_{\nu}}\right\|_{L^{2}(\Omega)}\right)\left\|z_{\vartheta_{k}}\right\|_{L^{2}(\Omega)} \\
& \leq \frac{c}{k}\left\|z_{u_{k}-u_{\nu}}\right\|_{L^{2}(\Omega)}^{2},
\end{aligned}
$$

where we have used (3.27) and (3.28). For the other part of the integrand, we find

$$
\begin{aligned}
\int_{\Omega} \mid \varphi_{\vartheta_{k}} & \frac{\partial^{2} a}{\partial y^{2}}\left(x, y_{\vartheta_{k}}\right) z_{\vartheta_{k}}^{2}-\varphi_{\nu} \frac{\partial^{2} a}{\partial y^{2}}\left(x, y_{\nu}\right) z_{u_{k}-u_{\nu}}^{2} \mid d x \\
& \leq \int_{\Omega}\left|\varphi_{\vartheta_{k}}-\varphi_{\nu}\right|\left|\frac{\partial^{2} a}{\partial y^{2}}\left(x, y_{\vartheta_{k}}\right)\right| z_{\vartheta_{k}}^{2} d x+\mathrm{II}+\mathrm{III},
\end{aligned}
$$

where

$$
\begin{aligned}
\mathrm{II} & :=\int_{\Omega}\left|\varphi_{\nu}\right|\left|\frac{\partial^{2} a}{\partial y^{2}}\left(x, y_{\vartheta_{k}}\right)-\frac{\partial^{2} a}{\partial y^{2}}\left(x, y_{\nu}\right)\right| z_{\vartheta_{k}}^{2} d x, \\
\mathrm{III} & :=\int_{\Omega}\left|\varphi_{\nu}\right|\left|\frac{\partial^{2} a}{\partial y^{2}}\left(x, y_{\nu}\right)\right|\left|z_{\vartheta_{k}}^{2}-z_{u_{k}-u_{\nu}}^{2}\right| d x .
\end{aligned}
$$

To further estimate the terms (3.30)-(3.32), we subtract the equations

$$
\begin{aligned}
A^{*} \varphi_{\vartheta_{k}}+\frac{\partial a}{\partial y}\left(x, y_{\vartheta_{k}}\right) \varphi_{\vartheta_{k}} & =y_{\vartheta_{k}}-y_{d}+\mu_{\nu}, \\
A^{*} \varphi_{\nu}+\frac{\partial a}{\partial y}\left(x, y_{\nu}\right) \varphi_{\nu} & =y_{\nu}-y_{d}+\mu_{\nu}
\end{aligned}
$$

and get with some intermediate function $\hat{y}_{\vartheta_{k}}$

$$
A^{*}\left(\varphi_{\vartheta_{k}}-\varphi_{\nu}\right)+\frac{\partial a}{\partial y}\left(x, y_{\nu}\right)\left(\varphi_{\vartheta_{k}}-\varphi_{\nu}\right)=y_{\vartheta_{k}}-y_{\nu}-\frac{\partial^{2} a}{\partial y^{2}}\left(x, \hat{y}_{\vartheta_{k}}\right)\left(y_{\vartheta_{k}}-y_{\nu}\right) \varphi_{\vartheta_{k}} .
$$

Therefore, we can estimate $\varphi_{\vartheta_{k}}-\varphi_{\nu}$ by

$$
\begin{aligned}
\left\|\varphi_{\vartheta_{k}}-\varphi_{\nu}\right\|_{L^{\infty}(\Omega)} & \leq c\left(\left\|y_{\vartheta_{k}}-y_{\nu}\right\|_{L^{2}(\Omega)}+\left\|y_{\vartheta_{k}}-y_{\nu}\right\|_{L^{\infty}(\Omega)}\left\|\varphi_{\vartheta_{k}}\right\|_{L^{2}(\Omega)}\right) \\
& \leq c\left\|u_{k}-u_{\nu}\right\|_{L^{2}(\Omega)} \leq \frac{c}{k} .
\end{aligned}
$$

For deriving the last line, we have considered the equations

$$
A y_{\vartheta_{k}}+a\left(x, y_{\vartheta_{k}}\right)=u_{\nu}+\vartheta_{k}\left(u_{k}-u_{\nu}\right) \quad \text { and } \quad A y_{\nu}+a\left(x, y_{\nu}\right)=u_{\nu} .
$$

Copyright ( by SIAM. Unauthorized reproduction of this article is prohibited. 
The estimates (3.28) and (3.33) imply

$$
\int_{\Omega}\left|\varphi_{\vartheta_{k}}-\varphi_{\nu}\right|\left|\frac{\partial^{2} a}{\partial y^{2}}\left(x, y_{\vartheta_{k}}\right)\right| z_{\vartheta_{k}}^{2} d x \leq \frac{c}{k}\left\|z_{u_{k}-u_{\nu}}\right\|_{L^{2}(\Omega)}^{2}
$$

Now we consider the terms II and III: From Assumption 2 and $y_{k}, y_{\nu} \in Y_{\gamma}$, we know that for every $\varepsilon>0$ there exists $\rho_{\varepsilon, \gamma}>0$ such that

$$
\left|\frac{\partial^{2} a}{\partial y^{2}}\left(x, y_{\vartheta_{k}}(x)\right)-\frac{\partial^{2} a}{\partial y^{2}}\left(x, y_{\nu}(x)\right)\right|<\varepsilon \quad \text { if } \quad\left|y_{\vartheta_{k}}(x)-y_{\nu}(x)\right|<\rho_{\varepsilon, \gamma} .
$$

Therefore, with (3.28) it holds that

$$
|\mathrm{II}| \leq\left\|\frac{\partial^{2} a}{\partial y^{2}}\left(\cdot, y_{\vartheta_{k}}\right)-\frac{\partial^{2} a}{\partial y^{2}}\left(\cdot, y_{\nu}\right)\right\|_{L^{\infty}(\Omega)}\left\|z_{u_{k}-u_{\nu}}\right\|_{L^{2}(\Omega)}^{2},
$$

where the $L^{\infty}$-norm above tends to zero as $k \rightarrow \infty$.

The estimate of III is an immediate consequence of (3.29). With all obtained estimates, we found

$$
\frac{\sigma}{2}\left\|u_{k}-u_{\nu}\right\|_{L^{2}(\Omega)}^{2} \leq \varepsilon_{k}\left\|z_{u_{k}-u_{\nu}}\right\|_{L^{2}(\Omega)}^{2} \leq C \varepsilon_{k}\left\|u_{k}-u_{\nu}\right\|_{L^{2}(\Omega)}^{2},
$$

where $\varepsilon_{k} \rightarrow 0$. This is only possible if $u_{k}=u_{\nu}$ holds for all sufficiently large $k$, which contradicts (3.21).

3.2. Case $\boldsymbol{\nu}=\mathbf{0}$. In this section, the functions $\mathcal{L}_{0}, J_{0}$, and $F_{0}$ are simply denoted by $\mathcal{L}, J$, and $F$, respectively. Let $\bar{u} \in U_{a d}$ be a control that satisfies the first-order necessary optimality system together with $(\bar{y}, \bar{\varphi}, \bar{\lambda}, \bar{\mu})$. For $\nu=0$, the second-order conditions (3.16)-(3.18) are not equivalent. The issue is to find out if any of these three conditions is sufficient for local optimality of $\bar{u}$. The assumption (3.16) is too weak; see [17] for an example. The condition (3.17) is too strong and it is never fulfilled when $\nu=0$; see [4]. The correct assumption is (3.18), as stated in the next theorem.

THEOREM 3.3. If the second-order condition (3.18) is satisfied, then there exist $\varepsilon>0$ and $\delta>0$ such that

$$
J(\bar{u})+\frac{\delta}{2}\left\|z_{u-\bar{u}}\right\|_{L^{2}(\Omega)}^{2} \leq J(u) \quad \forall u \in U_{a d} \text { with }\|u-\bar{u}\|_{L^{2}(\Omega)}<\varepsilon .
$$

The proof of this theorem follows the lines of the proof of Theorem 3.2 just changing $u_{k}-\bar{u}$ by $z_{u_{k}-\bar{u}}$ when necessary. For instance, (3.21) must be substituted by

$$
\left\|u_{k}-\bar{u}\right\|_{L^{2}(\Omega)}<\frac{1}{k} \text { and } J\left(u_{k}\right)<J\left(u_{\nu}\right)+\frac{1}{2 k}\left\|z_{u_{k}-\bar{u}}\right\|_{L^{2}(\Omega)}^{2} .
$$

The inequality (3.25) has to be replaced by

$$
\frac{1}{2} \frac{\partial^{2} \mathcal{L}}{\partial u^{2}}\left(u_{k}+\vartheta_{k}\left(u_{k}-\bar{u}\right), \bar{\mu}\right)\left(u_{k}-\bar{u}\right)^{2} \geq \frac{\sigma}{2}\left\|z_{u_{k}-\bar{u}}\right\|_{L^{2}(\Omega)}^{2}+\mathrm{I}
$$

The final contradiction admits the form

$$
\frac{\sigma}{2}\left\|z_{u_{k}-\bar{u}}\right\|_{L^{2}(\Omega)}^{2} \leq \varepsilon_{k}\left\|z_{u_{k}-\bar{u}}\right\|_{L^{2}(\Omega)}^{2} .
$$

Copyright (c) by SIAM. Unauthorized reproduction of this article is prohibited. 
COROLlary 3.4. Under the assumptions of Theorem 3.3, there exists $\bar{\delta}>0$ such that

$$
J(\bar{u})+\frac{\bar{\delta}}{2}\left\|y_{u}-\bar{y}\right\|_{L^{2}(\Omega)}^{2} \leq J(u) \quad \forall u \in U_{a d} \cap B_{\varepsilon}(\bar{u}) .
$$

This is an immediate consequence of Theorem 3.3. It is enough to use that

$$
\left\|y_{u}-\bar{y}\right\|_{L^{2}(\Omega)} \leq c\left\|z_{u-\bar{u}}\right\|_{L^{2}(\Omega)}
$$

and to take $\bar{\delta}=\delta / c^{2} ;$ cf. Casas [4].

4. Stability analysis with respect to $\nu$. In this section, we investigate the behavior of a sequence of optimal controls $u_{\nu}$ of $\left(\mathrm{P}_{\nu}\right)$ as $\nu \rightarrow 0$. Under natural assumptions, we expect the convergence $u_{\nu} \rightarrow u_{0}$, where $u_{0}$ is an optimal solution of $\left(\mathrm{P}_{0}\right)$. We recall our notation $u_{0}:=\bar{u}$ and $y_{0}:=\bar{y}$.

Let now $\left\{u_{\nu}\right\}_{\nu>0}$ be a sequence of solutions to $\left(\mathrm{P}_{\nu}\right)$, where $\nu=1 / k$ with $k \in \mathbb{N}$. By the boundedness of $\mathcal{U}_{\alpha, \beta}$, we can assume without loss of generality that $u_{\nu}$ converges weakly* in $L^{\infty}(\Omega)$ to some $\bar{u} \in \mathcal{U}_{\alpha, \beta}$. Therefore, we also have $y_{\nu} \rightarrow \bar{y}$ in $H_{0}^{1}(\Omega) \cap C_{0}(\bar{\Omega})$.

THEOREM 4.1.

(i) The weak ${ }^{*}$-limit $\bar{u}$ of $u_{\nu}$ is a solution to $\left(\mathrm{P}_{0}\right)$.

(ii) The sequence $\left\{u_{\nu}\right\}$ converges strongly in $L^{2}(\Omega)$.

Proof. For all $u \in U_{a d}$, there holds

$$
J(\bar{u}) \leq \liminf _{\nu \rightarrow 0} J\left(u_{\nu}\right) \leq \liminf _{\nu \rightarrow 0} J_{\nu}\left(u_{\nu}\right) \leq \limsup _{\nu \rightarrow 0} J_{\nu}\left(u_{\nu}\right) \leq \limsup _{\nu \rightarrow 0} J_{\nu}(u)=J(u) .
$$

Because $\bar{u}$ obviously belongs to $U_{a d}$, this shows that $\bar{u}$ solves $\left(\mathrm{P}_{0}\right)$ and hence (i) is proved.

Let us now show (ii). From

$$
J(\bar{u})+\frac{\nu}{2}\left\|u_{\nu}\right\|_{L^{2}(\Omega)}^{2} \leq J\left(u_{\nu}\right)+\frac{\nu}{2}\left\|u_{\nu}\right\|_{L^{2}(\Omega)}^{2}=J_{\nu}\left(u_{\nu}\right) \leq J_{\nu}(\bar{u})=J(\bar{u})+\frac{\nu}{2}\|\bar{u}\|_{L^{2}(\Omega)}^{2},
$$

it follows that

$$
\left\|u_{\nu}\right\|_{L^{2}(\Omega)} \leq\|\bar{u}\|_{L^{2}(\Omega)} \quad \forall \nu>0 .
$$

On the other hand, we find

$$
\|\bar{u}\|_{L^{2}(\Omega)} \leq \liminf _{\nu \rightarrow 0}\left\|u_{\nu}\right\|_{L^{2}(\Omega)} \leq \limsup _{\nu \rightarrow 0}\left\|u_{\nu}\right\|_{L^{2}(\Omega)} \leq\|\bar{u}\|_{L^{2}(\Omega)},
$$

where (4.1) was used in the last inequality. This shows the convergence of norms $\left\|u_{\nu}\right\|_{L^{2}(\Omega)} \rightarrow\|\bar{u}\|_{L^{2}(\Omega)}$ that, together with the weak convergence $u_{\nu} \rightarrow \bar{u}$ in $L^{2}(\Omega)$, yields the strong convergence in $L^{2}(\Omega)$. This shows II.

Let us prove a converse result.

THEOREM 4.2. Let $\bar{u}$ be a strict local minimum of $\left(\mathrm{P}_{0}\right)$ in the $L^{2}(\Omega)$ sense. Then, there exists a sequence $\left\{u_{\nu}\right\}_{\nu>0}$ of local minima of problems $\left(\mathrm{P}_{\nu}\right)$ such that $u_{\nu} \rightarrow \bar{u}$ strongly in $L^{2}(\Omega)$.

Proof. Since $\bar{u}$ is a strict local minimum of $\left(\mathrm{P}_{0}\right)$, there exists $\varepsilon>0$ such that $\bar{u}$ is the unique solution of

$$
\left(\mathrm{P}_{\varepsilon}\right) \quad \min _{u \in U_{a d} \cap \bar{B}_{\varepsilon}(\bar{u})} J(u),
$$

Copyright (c) by SIAM. Unauthorized reproduction of this article is prohibited. 
where $\bar{B}_{\varepsilon}(\bar{u})$ denotes the $L^{2}(\Omega)$-closed ball with center at $\bar{u}$ and radius $\varepsilon$. Analogously, for every $\nu>0$ we consider the problems

$$
\left(\mathrm{P}_{\nu, \varepsilon}\right) \quad \min _{u \in U_{a d} \cap \bar{B}_{\varepsilon}(\bar{u})} J_{\nu}(u) .
$$

For every $\nu>0$ there exists at least one solution $u_{\nu}$ of $\left(\mathrm{P}_{\nu, \varepsilon}\right)$. We can apply Theorem 4.1 and deduce that, for a subsequence if necessary, $\left\{u_{\nu}\right\}$ converges strongly in $L^{2}(\Omega)$ to a solution of $\left(\mathrm{P}_{\varepsilon}\right)$. But the only solution of $\left(\mathrm{P}_{\varepsilon}\right)$ is $\bar{u}$, and hence the whole sequence $\left\{u_{\nu}\right\}$ converges strongly to $\bar{u}$ in $L^{2}(\Omega)$. For $\nu$ sufficiently small, $\left\|u_{\nu}-\bar{u}\right\|_{L^{2}(\Omega)}<\varepsilon$, and consequently $u_{\nu}$ is a local solution of $\left(\mathrm{P}_{\nu}\right)$.

Assumption 4 (linearized slater condition). There exists $u_{s} \in \mathcal{U}_{\alpha, \beta}$ such that

$$
\left|\left(\bar{y}+z_{u_{s}-\bar{u}}\right)(x)\right|<\gamma \quad \forall x \in \Omega,
$$

where $z_{u_{s}-\bar{u}}=G^{\prime}(\bar{u})\left(u_{s}-\bar{u}\right)$. In what follows, we write for short $z_{\nu, s}:=z_{\nu, u_{s}-u_{\nu}}$.

TheOrem 4.3. Under Assumption 4, there exists some $\nu_{s}>0$ such that

$$
\left|\left(y_{\nu}+z_{\nu, s}\right)(x)\right|<\gamma \quad \forall x \in \Omega \quad \forall \nu<\nu_{s},
$$

where $z_{\nu, u_{s}-u_{\nu}}$ is the solution to

$$
\left\{\begin{aligned}
A z+\frac{\partial a}{\partial y}\left(x, y_{\nu}\right) z & =u_{s}-u_{\nu} & & \text { in } \Omega \\
z & =0 & & \text { on } \Gamma .
\end{aligned}\right.
$$

Proof. The sequence $\left\{y_{\nu}\right\}_{\nu}$ converges to $\bar{y}$, uniformly in $\Omega$. Therefore, it suffices to prove that $\left\|z_{\nu, s}-z_{u_{s}-u_{\nu}}\right\|_{C_{0}(\Omega)} \rightarrow 0$ for $\nu \rightarrow 0$.

Subtracting the equations for $z_{\nu, s}$ and $z_{u_{s}-u_{\nu}}$, we obtain with some $\vartheta_{k} \in(0,1)$

$$
\begin{aligned}
& A\left(z_{\nu, s}-z_{u_{s}-\bar{u}}\right)+\frac{\partial a}{\partial y}(x, \bar{y})\left(z_{\nu, s}-z_{u_{s}-\bar{u}}\right) \\
& \quad=-\frac{\partial^{2} a}{\partial y^{2}}\left(x, \bar{y}+\vartheta_{k}\left(y_{\nu}-\bar{y}\right)\right)\left(y_{\nu}-\bar{y}\right) z_{\nu, s}+\bar{u}-u_{\nu} .
\end{aligned}
$$

This implies

$\left\|z_{\nu, s}-z_{u_{s}-u_{\nu}}\right\|_{C_{0}(\Omega)} \leq C\left(\left\|y_{\nu}-y_{\bar{u}}\right\|_{C_{0}(\Omega)}\left\|z_{\nu, s}\right\|_{L^{2}(\Omega)}+\left\|\bar{u}-u_{\nu}\right\|_{L^{2}(\Omega)}\right) \rightarrow 0$ if $\nu \rightarrow 0$.

Notice that $\left\|z_{\nu, s}\right\|_{L^{2}(\Omega)}$ is obviously bounded.

This result is the basis for proving the following one.

TheOREM 4.4. Under Assumption 4, for all $\nu<\nu_{s}$ there exist $\mu_{\nu} \in M(\Omega)$, $\lambda_{\nu} \in L^{2}(\Omega)$, and $\varphi_{\nu} \in W^{1, s}(\Omega)$ for all $s<n /(n-1)$ such that

$$
\begin{array}{clrl}
A^{*} \varphi_{\nu}+\frac{\partial a}{\partial y}\left(x, y_{\nu}\right) \varphi_{\nu} & =y_{\nu}-y_{d}+\mu_{\nu} & & \text { in } \Omega, \\
\varphi_{\nu} & =0 & & \text { on } \Gamma, \\
\int_{\Omega}\left(y-y_{\nu}\right) d \mu_{\nu} \leq 0 & \forall y \in Y_{\nu}, & & \\
\int_{\Omega} \lambda_{\nu}\left(u-u_{\nu}\right) d x+\int_{\Omega}\left|u_{\nu}\right| d x \leq \int_{\Omega}|u| d x & & \forall u \in L^{1}(\Omega), \\
\int_{\Omega}\left(\varphi_{\nu}+\nu u_{\nu}+\kappa \lambda_{\nu}\right)\left(u-u_{\nu}\right) d x \geq 0 & \forall u \in \mathcal{U}_{\alpha, \beta} .
\end{array}
$$

Copyright $@$ ( ) by SIAM. Unauthorized reproduction of this article is prohibited. 
Moreover, there is some $\bar{\mu}$ in $M(\Omega)$ such that, for $\nu \rightarrow 0$,

$$
\begin{aligned}
\mu_{\nu} & * \bar{\mu} \text { in } M(\Omega) \text { and }\left\|\mu_{\nu}\right\|_{M(\Omega)} \rightarrow\|\bar{\mu}\|_{M(\Omega)}, \\
y_{\nu} & \rightarrow \bar{y} \text { in } H_{0}^{1}(\Omega) \cap C(\bar{\Omega}), \\
\varphi_{\nu} & \rightarrow \bar{\varphi} \text { in } W^{1, s}(\Omega), \\
\lambda_{\nu} & \rightarrow \bar{\lambda} \text { in } L^{2}(\Omega)
\end{aligned}
$$

(all limits for subsequences, if necessary). Finally, $(\bar{u}, \bar{y}, \bar{\varphi}, \bar{\mu}, \bar{\lambda})$ satisfies the optimality system of $\left(\mathrm{P}_{0}\right)$.

Proof. The relations (4.4)-(4.7) constitute the necessary optimality conditions for the problem $\left(\mathrm{P}_{\nu}\right)$, which follow from the linearized Slater condition. Therefore, it remains to show the convergence properties.

(i) Boundedness of $\left\{\left(u_{\nu}, y_{\nu}, \varphi_{\nu}, \lambda_{\nu}, \mu_{\nu}\right)\right\}_{\nu>0}$ : The boundedness of $\left\{\left(u_{\nu}, y_{\nu}\right)\right\}$ in $L^{\infty}(\Omega) \times\left[H_{0}^{1}(\Omega) \cap C(\bar{\Omega})\right]$ is obvious. The identity (2.14) shows the boundedness of $\left\{\lambda_{\nu}\right\}$ in $L^{\infty}(\Omega)$. If we prove the boundedness of $\left\{\mu_{\nu}\right\}$, then the boundedness of $\left\{\varphi_{\nu}\right\}$ follows from (2.8). Let us study the sequence $\left\{\mu_{\nu}\right\}$. Let $\nu_{s}>0$ be as defined in Theorem 4.3.

Inequality (4.5) can be written

$$
\gamma\left\|\mu_{\nu}\right\|_{M(\Omega)}=\sup _{y \in Y_{\gamma}} \int_{\Omega} y d \mu_{\nu}=\int_{\Omega} y_{\nu} d \mu_{\nu} \quad \forall \nu<\nu_{s} .
$$

From the convergence $\left(y_{\nu}, z_{\nu, s}\right) \rightarrow\left(\bar{y}, z_{u_{s}-\bar{u}}\right)$ in $C_{0}(\Omega) \times C_{0}(\Omega)$, we deduce the existence of $\nu_{0} \in\left(0, \nu_{s}\right]$ such that

$$
\rho_{\nu}=\gamma-\left\|y_{\nu}+z_{\nu, s}\right\|_{C_{0}(\Omega)} \geq \frac{\rho}{2}=\frac{1}{2}\left(\gamma-\left\|\bar{y}+z_{u_{s}-\bar{u}}\right\|_{C_{0}(\Omega)}\right) \quad \forall \nu<\nu_{0} .
$$

Therefore, there holds

$$
y_{\nu}+z_{\nu, s}+z \in Y_{\gamma} \quad \forall z \in \bar{B}_{\rho / 2}(0) \subset C_{0}(\Omega) \text { and } \forall \nu<\nu_{0} .
$$

From this and (4.12), we deduce

$$
\int_{\Omega}\left(z_{\nu, s}+z\right) d \mu_{\nu}=\int_{\Omega}\left[\left(y_{\nu}+z_{\nu, s}+z\right)-y_{\nu}\right] d \mu_{\nu} \leq 0 \quad \forall z \in \bar{B}_{\rho / 2}(0) \quad \forall \nu<\nu_{0},
$$

and hence

$$
\frac{\rho}{2}\left\|\mu_{\nu}\right\|_{M(\Omega)}=\sup _{z \in \bar{B}_{\rho / 2}(0)} \int_{\Omega} z d \mu_{\nu} \leq-\int_{\Omega} z_{\nu, s} d \mu_{\nu} \quad \forall \nu<\nu_{0} .
$$

From (3.2) and (3.5), we get

$$
F_{\nu}^{\prime}\left(u_{\nu}\right)\left(u-u_{\nu}\right)+\kappa \int_{\Omega} \lambda_{\nu}\left(u-u_{\nu}\right) d x+\int_{\Omega} z_{\nu, u-u_{\nu}} d \mu_{\nu} \geq 0 \quad \forall u \in \mathcal{U}_{\alpha, \beta} .
$$

Now, taking $u=u_{s}$, we find

$$
-\int_{\Omega} z_{\nu, s} d \mu_{\nu} \leq F_{\nu}^{\prime}\left(u_{\nu}\right)\left(u_{s}-u_{\nu}\right)+\kappa \int_{\Omega} \lambda_{\nu}\left(u_{s}-u_{\nu}\right) d x \leq C \quad \forall \nu<\nu_{0} .
$$

By (4.13), this implies the desired boundedness of $\left\{\mu_{\nu}\right\}$. Therefore, taking a subsequence, if necessary, we have that $\mu_{\nu} \stackrel{*}{\rightarrow} \bar{\mu}$ in $M(\Omega)$.

Copyright (C) by SIAM. Unauthorized reproduction of this article is prohibited. 
(ii) Convergence in the optimality system: Passing to the limit $\nu \rightarrow 0$ in (4.5) and using that $y_{\nu} \rightarrow \bar{y}$ in $C_{0}(\Omega)$, it follows that

$$
\int_{\Omega}(y-\bar{y}) d \bar{\mu} \leq 0 \quad \forall y \in Y_{\gamma} .
$$

This shows that $\bar{\mu}$ is a Lagrange multiplier for $\left(\mathrm{P}_{0}\right)$. Moreover, from (4.12), we get

$$
\gamma\left\|\mu_{\nu}\right\|_{M(\Omega)}=\int_{\Omega} y_{\nu} d \mu_{\nu} \rightarrow \int_{\Omega} \bar{y} d \bar{\mu}=\gamma\|\bar{\mu}\|_{M(\Omega)},
$$

which completes the proof of (4.8).

It is known that for every $f \in W^{-1, s^{\prime}}(\Omega)$ with $s^{\prime}>n$, the solution $z$ of

$$
\begin{aligned}
A z+\frac{\partial a}{\partial y}\left(x, y_{\nu}\right) z & =f & & \text { in } \Omega, \\
z & =0 & & \text { on } \Gamma
\end{aligned}
$$

belongs to $C^{\theta}(\bar{\Omega})$ for some $0<\theta<1$. Moreover, $\|z\|_{C^{\theta}(\bar{\Omega})} \leq C\|f\|_{W^{-1, s^{\prime}}(\Omega)}$ holds, where $C$ is independent of $f$; see [23, Theorem 7.3]. Then, the compactness of the embedding $C^{\theta}(\bar{\Omega}) \cap C_{0}(\Omega) \hookrightarrow C_{0}(\Omega)$ implies that the linear mapping $f \mapsto z$ is compact from $W^{-1, s^{\prime}}(\Omega)$ to $C_{0}(\Omega)$. By transposition we obtain that the solution mapping associated with (4.4) is compact from $M(\Omega)$ to $W_{0}^{1, s}(\Omega)$. Hence, the convergence $\varphi_{\nu} \rightarrow \bar{\varphi}$ is strong in $W_{0}^{1, s}(\Omega)$. Since $W^{1, s}(\Omega)$ is continuously embedded in $L^{2}(\Omega)$ for $s$ close enough to $n /(n-1)$, we obtain by $(2.14)$ and $(2.20)$

$$
\left\|\lambda_{\nu}-\bar{\lambda}\right\|_{L^{2}(\Omega)} \leq \frac{1}{\kappa}\left\|\varphi_{\nu}-\bar{\varphi}\right\|_{L^{2}(\Omega)} \rightarrow 0, \quad \text { as } \nu \rightarrow 0 .
$$

Finally, it is easy to pass to the limit in (4.7). Thus, we have proved that $(\bar{u}, \bar{y}, \bar{\varphi}, \bar{\mu}, \bar{\lambda})$ obeys the optimality system of $\left(\mathrm{P}_{0}\right)$.

Corollary 4.5. For all $\nu<\nu_{s}$, the adjoint states $\varphi_{\nu}$ belong to $L^{\infty}(\Omega) \cap H_{0}^{1}(\Omega)$ and the estimates

$$
\left\|\varphi_{\nu}\right\|_{L^{\infty}(\Omega)}+\left\|\varphi_{\nu}\right\|_{H_{0}^{1}(\Omega)} \leq M
$$

are satisfied with some $M>0$. Therefore, $\bar{\varphi}$ obeys the same estimates also. Consequently, $\mu_{\nu} \rightarrow \bar{\mu}$ in $H^{-1}(\Omega)$ and $\varphi_{\nu} \rightarrow \bar{\varphi}$ in $H_{0}^{1}(\Omega)$.

This corollary is an obvious consequence of Theorems 2.8 and 4.4. Indeed, it is enough to observe that, according to (2.25)-(2.27) and the boundedness of $\left\{\left(u_{\nu}, y_{\nu}, \varphi_{\nu}, \lambda_{\nu}, \mu_{\nu}\right)\right\}_{\nu>0}$ established in the above theorem, $M_{\nu}$ is uniformly bounded by a constant $M$ independent of $\nu$.

Let us finally estimate the convergence rate for $y_{\nu} \rightarrow \bar{y}$ in $L^{2}(\Omega)$. For this purpose, we assume that $\bar{u}$ is a local solution of $\left(\mathrm{P}_{0}\right)$ that, together with some multiplier $\bar{\mu}$, fulfills the second-order sufficient optimality conditions (3.18). Therefore, the (generalized) quadratic growth condition

$$
J(\bar{u})+\frac{\bar{\delta}}{2}\left\|y_{u}-\bar{y}\right\|_{L^{2}(\Omega)}^{2} \leq J(u) \quad \forall u \in U_{a d} \cap B_{\varepsilon}(\bar{u})
$$

is satisfied with some positive $\bar{\delta}$ and $\varepsilon$; see Corollary 3.4 .

Copyright $@$ ㅇ by SIAM. Unauthorized reproduction of this article is prohibited. 
We now select a sequence $\left\{u_{\nu}\right\}$ of local solutions to $\left(\mathrm{P}_{\nu}\right)$ converging strongly in $L^{2}(\Omega)$ to $\bar{u}$. Thanks to Theorem 4.2 , this selection is possible. Next, we estimate with $(3.35)$

$$
\begin{aligned}
J(\bar{u})+\frac{\bar{\delta}}{2}\left\|y_{\nu}-\bar{y}\right\|_{L^{2}(\Omega)}^{2}+\frac{\nu}{2}\left\|u_{\nu}\right\|_{L^{2}(\Omega)}^{2} & \leq J\left(u_{\nu}\right)+\frac{\nu}{2}\left\|u_{\nu}\right\|_{L^{2}(\Omega)}^{2}=J_{\nu}\left(u_{\nu}\right) \\
& \leq J_{\nu}(\bar{u})=J(\bar{u})+\frac{\nu}{2}\|\bar{u}\|_{L^{2}(\Omega)}^{2} .
\end{aligned}
$$

Subtracting the term $J(\bar{u})$ from both ends of this chain of inequalities and using (4.1), we find

$$
\begin{aligned}
\left\|y_{\nu}-\bar{y}\right\|_{L^{2}(\Omega)}^{2} & \leq \frac{\nu}{\bar{\delta}}\left(\|\bar{u}\|_{L^{2}(\Omega)}^{2}-\left\|u_{\nu}\right\|_{L^{2}(\Omega)}^{2}\right) \\
& =\frac{\nu}{\bar{\delta}}\left(\|\bar{u}\|_{L^{2}(\Omega)}+\left\|u_{\nu}\right\|_{L^{2}(\Omega)}\right)\left(\|\bar{u}\|_{L^{2}(\Omega)}-\left\|u_{\nu}\right\|_{L^{2}(\Omega)}\right) \\
& \leq \frac{\nu}{\bar{\delta}}\left(\|\bar{u}\|_{L^{2}(\Omega)}+\left\|u_{\nu}\right\|_{L^{2}(\Omega)}\right)\left\|\bar{u}-u_{\nu}\right\|_{L^{2}(\Omega)} \\
& \leq \frac{2}{\bar{\delta}}\|\bar{u}\|_{L^{2}(\Omega)}\left\|\bar{u}-u_{\nu}\right\|_{L^{2}(\Omega)} \nu .
\end{aligned}
$$

Finally, this implies the convergence rate

$$
\lim _{\nu \rightarrow 0} \frac{\left\|y_{\nu}-\bar{y}\right\|_{L^{2}(\Omega)}}{\sqrt{\nu}}=0 .
$$

Acknowledgment. The authors are grateful to one of the referees who suggested an improvement with respect to the strong convergence stated in (4.10).

\section{REFERENCES}

[1] J.F. Bonnans and E. Casas, Contrôle de systèmes elliptiques semilinéaires comportant des contraintes sur l'état, in Nonlinear Partial Differential Equations and Their Applications, Collège de France Seminar, H. Brezis and J.L. Lions, eds., Longman Scientific \& Technical, Harlow, UK, 8 (1988), pp. 69-86.

[2] F. Bonnans and A. Shapiro, Perturbation Analysis of Optimization Problems, SpringerVerlag, New York, 2000.

[3] E. CASAS, Control of an elliptic problem with pointwise state constraints, SIAM J. Control Optim., 24 (1986), pp. 1309-1318.

[4] E. CASAS, Second order analysis for bang-bang control problems of PDEs, SIAM J. Control Optim., 50 (2012), pp. 2355-2372.

[5] E. Casas, C. Clason, and K. Kunisch, Approximation of elliptic control problems in measure spaces with sparse solutions, SIAM J. Control Optim., 50 (2012), pp. 1735-1752.

[6] E. Casas, C. Clason, and K. Kunisch, Parabolic control problems in measure spaces with sparse solutions, SIAM J. Control Optim., 51 (2013), pp. 28-63.

[7] E. Casas, R. Herzog, and G. Wachsmuth, Optimality conditions and error analysis of semilinear elliptic control problems with $L^{1}$ cost functional, SIAM J. Optim., 22 (2012), pp. 795820.

[8] E. Casas, R. Herzog, and G. Wachsmuth, Approximation of sparse controls in semilinear equations by piecewise linear functions, Numer. Math., 122 (2012), pp. 645-669.

[9] E. CASAS AND K. Kunisch, Optimal control of semilinear elliptic equations in measure spaces, SIAM J. Control Optim., 52 (2014), pp. 339-364.

[10] E. Casas, M. Mateos, and B. Vexler, New regularity results and improved error estimates for optimal control problems with state constraints, ESAIM Control Optim. Calc. Var., to appear.

[11] E. Casas, J.C. DE los Reyes, And F. Tröltzsch, Sufficient second order optimality conditions for semilinear control problems with pointwise state constraints, SIAM J. Optim., 19 (2008), pp. 616-643.

Copyright $@$ by SIAM. Unauthorized reproduction of this article is prohibited. 
[12] E. Casas, C. Ryll, And F. Tröltzsch, Sparse optimal control of the Schlögl and FitzHughNagumo systems, Comput. Methods Appl. Math., 13 (2013), pp. 415-442.

[13] E. Casas, F. Tröltzsch, And A. Unger, Second order sufficient optimality conditions for some state-constrained control problems of semilinear elliptic equations, SIAM J. Control Optim., 38 (2000), pp. 1369-1391.

[14] E. Casas And E. Zuazua, Spike controls for elliptic and parabolic PDE, Systems Control Lett., 62 (2013), pp. 311-318.

[15] F. Clarke, Optimization and Nonsmooth Analysis, John Wiley \& Sons, Toronto, 1983.

[16] G. Dal Maso, F. Murat, L. Orsina, and A. Prignet, Renormalized solutions of elliptic equations with general measure data, Ann. Sc. Norm. Super. Pisa Cl. Sci., 28 (1999), pp. $741-808$.

[17] J.C. DunN, On second order sufficient optimality conditions for structured nonlinear programs in infinite-dimensional function spaces, in Mathematical Programming with Data Perturbations, A. Fiacco, ed., Marcel Dekker, New York, 1998, pp. 83-107.

[18] D. Gilbarg And N.S. Trudinger, Elliptic Partial Differential Equations of Second Order, Springer-Verlag, New York, 1977.

[19] R. Herzog, G. Stadler, and G. Wachsmuth, Directional Sparsity in Optimal Control of Partial Differential Equations, SIAM J. Control Optim., 50 (2012), pp. 943-963.

[20] W. Littman, G. Stampacchia, and H.F. Weinberger, Regular points for elliptic equations with discontinuous coefficients, Ann. Sc. Norm. Sup. Pisa, 17 (1963), pp. 43-77.

[21] K. Pieper and B. Vexler, A priori error analysis for discretization of sparse elliptic optimal control problems in measure space, SIAM J. Control Optim., 51 (2013), pp. 2788-2808.

[22] G. Stadler, Elliptic optimal control problems with $L^{1}$-control cost and applications for the placement of control devices, Comput. Optim. Appl., 444 (2009), pp. 159-181.

[23] G. Stampacchia, Le problème de Dirichlet pour les équations elliptiques du second ordre à coefficients discontinus, Ann. Inst. Fourier (Grenoble), 15 (1965), pp. 189-258.

[24] F. Tröltzsch, Optimal Control of Partial Differential Equations: Theory, Methods and Applications, Vol. 112, AMS, Providence, RI, 2010.

[25] G. Wachsmuth And D. Wachsmuth, Convergence and regularisation results for optimal control problems with sparsity functional, ESAIM Control Optim. Calc. Var., 17 (2011), pp. 858-886.

Copyright (c) by SIAM. Unauthorized reproduction of this article is prohibited. 